\title{
Population Responses Represent Vocalization Identity, Intensity, and Signal-to-Noise Ratio in Primary Auditory Cortex
}

\author{
Abbreviated title: Population Coding of Vocalization in Noise \\ Ruiye $\mathrm{Ni}^{1}$, David A. Bender ${ }^{2}$, Dennis L. Barbour ${ }^{1}$ \\ 1. Laboratory of Sensory Neuroscience and Neuroengineering \\ Department of Biomedical Engineering \\ Washington University in St. Louis \\ St. Louis, Missouri 63130, U.S.A. \\ 2. Department of Biology \\ Washington University in St. Louis \\ St. Louis, Missouri 63130, U.S.A.
}

\section{Please send correspondence to: \\ Dr. Dennis L. Barbour \\ Department of Biomedical Engineering \\ Washington University in St. Louis \\ One Brookings Dr., Campus Box 1097 \\ Uncas Whitaker Hall Room 200E \\ St. Louis, MO 63130, U.S.A. \\ Tel. (314) 935-7548, Fax. (314) 935-7448 \\ Email: dbarbour@biomed.wustl.edu}

Number of Pages: 44

Number of Figures: 15

Number of Abstract Words: 217

Number of Introduction Words: 700

Number of Discussion Words: 787

The authors declare no competing financial interests.

Acknowledgment:

This work was supported by the National Institutes of Health grant R01-DC009215. We thank Kim Kocher for valuable assistance with animal training and data collection. Our thanks also go to Wensheng Sun for her help with neurophysiology experiment preparation. 


\section{Abstract [217/250 words]}

45 The ability to process speech signals under challenging listening environments is critical

46 for speech perception. Great efforts have been made to reveal the underlying single unit

47 encoding mechanism. However, big variability is usually discovered in single-unit responses,

48 and the population coding mechanism is yet to be revealed. In this study, we are aimed to study

49 how a population of neurons encodes behaviorally relevant signals subjective to change in

50 intensity and signal-noise-ratio (SNR). We recorded single-unit activity from the primary

51 auditory cortex of awake common marmoset monkeys (Callithrix jacchus) while delivering

52 conspecific vocalizations degraded by two different background noises: broadband white noise

53 (WGN) and vocalization babble (Babble). By pooling all single units together, the pseudo-

54 population analysis showed the population neural responses track intra- and inter-trajectory angle

55 evolutions track vocalization identity and intensity/SNR, respectively. The ability of the

56 trajectory to track the vocalizations attribute was degraded to a different degree by different

57 noises. Discrimination of neural populations evaluated by neural response classifiers revealed

58 that a finer optimal temporal resolution and longer time scale of temporal dynamics were needed

59 for vocalizations in noise than vocalizations at multiple different intensities. The ability of

60 population responses to discriminate between different vocalizations were mostly retained above

61 the detection threshold.

62 Key Words: auditory cortex, marmoset monkeys, vocalizations, noise interference, intensity,

63 signal-to-noise ratio, population coding 


\section{Significance Statement [83/120 words]}

65 How our brain excels in the challenge of precise acoustic signal encoding against noisy

66 environment is of great interest for scientists. Relatively few studies have strived to tackle this

67 mystery from the perspective of neural population responses. Population analysis reveals the

68 underlying neural encoding mechanism of complex acoustic stimuli based upon a pool of single

69 units via vector coding. We suggest the spatial population response vectors as one important way

70 for neurons to integrate multiple attributes of natural acoustic signals, specifically, marmots'

71 vocalizations. 


\section{Introduction [700/650 words]}

Due to the inherent noise in the activity of individual neurons, multiple presentations of an identical sensory stimulus do not yield exactly the same spike trains. By computing the spiking rate averaged across trials to get rid of the response noisiness, researchers have generally expected to estimate the true firing rate driven by a stimulus. Large amounts of single-unit analysis have been conducted in this fashion. In studying neural responses to auditory stimuli, much insight has been gained from analyzing coding properties of individual neurons and at a population level that averaged across individual cells, based upon the simplistic rate-coding

81 hypothesis (Aitkin et al. 1986; Barbour 2011; Bendor and Wang 2005; Imig et al. 1990; Woolley typically been overlooked. This seems to be a minor concern for studies investigating relatively

84 simple acoustic stimuli, such as pure tones, but there is study showing that even stationary acoustic stimuli induce dynamic responses on a population level. For more complicated acoustic signals with rich temporal-spectral structures, for instance, marmoset vocalizations (Gehr et al.

87 2000; Nagarajan et al. 2002), an analytical method for inspecting the response properties among populations is needed.

In contrast to single-unit coding, population coding hypothesizes that the stimulus

90 information is encoded in the brain by a large population of neurons via distributed firing rate

91 patterns (McIlwain 2001). Over the past two decades, multiple population analyses have

92 emerged to reveal the neural encoding and decoding properties at the population level, such as

93 population variability analysis and spatiotemporal coding analysis (Churchland et al. 2010).

94 More importantly, to study the dynamics of population variability in response to vocalizations at

95 multiple intensifies and SNR, this paper further asks whether acoustic features of vocalizations 

structures. In the sensory areas, even presentation of a temporally unstructured stimulus, such as

99 a stationary odor or pure tone, is likely to induce a complex temporal pattern of spiking (Bartho

100 et al. 2009; Stopfer et al. 2003). By unifying the temporally-structured responses of individual

101 neurons, we can visualize the complex spatiotemporal patterns at the population level. The

102 spatiotemporal patterns of the population responses vary with the stimulus when a particular

103 feature of the stimulus is slightly changed, such as intensity (Stopfer et al. 2003). Such

104 visualization analysis has revealed the dynamics of responses to relative simple stimuli, however,

105 little is known about the spatiotemporal patterns of complex vocalization stimuli. Here, by

106 varying intensities and SNR levels, the alteration of spatiotemporal patterns of population neural

107 responses to five marmoset conspecific vocalizations was studied and the hypothesis that the

108 population responses are not just a linear scaling of their amplitude was tested.

Neurometric analysis is a useful tool for linking neural activity with perception to

110 identify the underlying neural substrate that generates the sensory perception and behaviors of

111 interest (Walker et al., 2008). Individual neurons vary widely in their ability to discriminate

112 complex acoustic stimuli (Narayan et al. 2006; Schneider and Woolley 2010; Wang et al. 2007).

113 However, the degree to which a population of neurons can recognize different types of

114 vocalizations at multiple intensities and SNR levels is not well known. Would distributed firing

115 rate patterns across a whole population of recorded cells optimize the performance of the

116 stimulus discrimination task, or is there a subpopulation of neurons that yields the best

117 performance? With respect to the dynamics along the time course, does a population of neurons

118 have a constant discriminability, or are the neural responses at certain time epochs better than at

119 others? These questions were investigated by building population response decoding models that 
120 are sensitive to temporal discharge patterns. Using this decoding tool, we further inferred the

121 perception intensity threshold of vocalizations and the detection threshold of vocalizations

122 masked with WGN/Babble noise.

123 In summary, in this paper, by pooling the activities of individual neurons in response to

124 vocalizations at multiple intensities and SNR levels, we analyzed population responses from

125 three aspects: population response variability with respect to time, the spatiotemporal structures

126 of population responses, and the ability of population responses to identify stimuli under

127 different experimental conditions. 


\section{Materials and Methods}

\section{Surgery and recording}

131 All training, recording, and surgical procedures comply with US National Institute of

132 Health Guide for the Care and Use of Laboratory Animals and are approved by the Washington

133 University in St. Louis Animal Studies Committee.

The experimental materials and procedures were similar to those reported previously

135 (Bender et al. 2016; Ni et al. 2016). Adult common marmoset monkeys (Callithrix jacchus) were

used as subjects. Subjects were trained to sit upright in a custom, minimally restraining primate

137 chair inside a double walled sound-attenuation booth (IAC 120a-3, Bronx, NY) while their heads

138 were fixed in place by head posts for the same length of duration as that would be for physiology

139 recording. After they became used to this setup, a custom head cap for recording was surgically

140 affixed to the skull of each subject, and temporalis muscle was removed during surgery. The

141 location of the vasculature running within the lateral sulcus was marked on the skull. According

142 to the landmark, microcraniotomies $(<1 \mathrm{~mm}$ diameter) were drilled through the skull with a

143 custom drill directly over auditory cortex. The location of A1 was identified anatomically based

144 upon lateral sulcus and bregma landmarks and confirmed with physiological mapping (Stephan

145 et al. 2012). Before experiments, the animals were allowed to take sufficient time to recover

146 following the surgery.

147 A single high-impedance tungsten-epoxy $125 \mu \mathrm{m}$ electrode $(\sim 5 \mathrm{M} \Omega @ 1 \mathrm{kHz}, \mathrm{FHC}$,

148 Bowdoin, ME) was advanced perpendicularly to the cortical surface within the

149 microcraniotomies. Microelectrode signals were amplified using an AC differential amplifier

150 (AM systems 1800, Sequim, WA) with the differential lead attached to a grounding screw.

151 Single-unit action potentials were sorted online using manual template-based spike-sorting

152 hardware and software (Alpha Omega, Nazareth, Israel). When a template match occurred, the 
153 spike-sorting hardware relayed a TTL pulse to DSP system (TDTRX6, Alachua FL) that

154 temporally aligned recorded spike times $(2.5 \mu$ s accuracy) with stimulus delivery. The recording

155 locations within the head cap were varied daily, covering all the areas of interest.

Acoustic stimulation

Two types of noises, white Gaussian noise (WGN) and 4-vocalization babble noise (Babble), were mixed with five natural marmoset conspecific-vocalizations from distinct

159 acoustic classes (Trill-Phee, Peep-Trill, Trill-Twitter, Tsik-String and Peep-String) to generate

160 noisy vocalizations at eight different signal-to-noise ratios (SNR; -15 dB SPL to $20 \mathrm{~dB}$ SPL with

$1615 \mathrm{~dB}$ SPL interval, plus pure noise and clean vocalization). Spectral power was normalized at

162 each SNR level. The five calls were selected to represent most of the acoustic features of a set of

163 twenty calls. In order to distinguish the onset responses induced by the components of noise and

164 vocalization in the synthesized stimuli, two $250 \mathrm{~ms}$ intervals of pure noise were concatenated to

165 both ends of each noisy vocalization. Babble noise was created by shuffling superimposed 50

166 ms-long pieces of four vocalizations, which were different from the five test vocalizations. Both

167 WGN and babble were synthesized with duration equivalent to the longest vocalization, the Trill-

168 Phee. For the other four vocalizations, WGN and babble noise were truncated to the same length

169 as each vocalization.

\section{Experimental procedure}

Single-unit activities in auditory cortex were recorded from two alert adult marmoset

172 monkeys while they were passively listening to the playback of natural and synthesized

173 conspecific vocalizations. Auditory neurons were detected based upon their responses evoked by

174 pure tones and vocalizations. Once an auditory neuron was isolated, its characteristic frequency

175 was estimated by using random spectrum stimuli (Barbour and Wang 2003) and/or pure tones. 
176 Next, its rate level functions of five vocalizations were measured with attenuations from $30 \mathrm{~dB}$ to

$17790 \mathrm{~dB}$ with $20 \mathrm{~dB}$ step in random order with 10 repetitions. The attenuation evoking strong

178 responses to most of the vocalizations was selected to deliver noisy vocalizations. Degraded

179 versions' of five vocalizations were also randomly displayed with repetitions between 5 and 10.

\section{Analysis}

181 The dataset is the recorded single-unit responses to five vocalizations (Trillphee,

182 Peeptrill, Trilltwitter, Tsikstring, and Peepstring) at four intensities (from $15 \mathrm{~dB}$ SPL to $75 \mathrm{~dB}$

183 SPL, in $20 \mathrm{~dB}$ SPL steps). In total, $N=326$ single units were included in the analysis.

184 For each unit of both datasets, a peristimulus time histogram (PSTH) for each response

185 trial was calculated by binning spike trains into rate vectors with a $50 \mathrm{~ms}$ window in $10 \mathrm{~ms}$ steps.

186 The following data analyses are based upon this preprocessing, unless otherwise stated. All data

187 analyses were conducted in MATLAB R2014a (The MathWorks Inc, Natick, MA). Because

188 most of the neurons in our dataset were recorded sequentially one at a time, we created pseudo-

189 populations to substitute for simultaneous recordings. Creating these pseudo-populations

190 potentially ignores the correlation between individual neurons that exists in a simultaneously

191 recorded neuronal population, and may change the estimates of the absolute level of

192 performance. The majority of conclusions drawn in this study, however, would most likely not

193 be altered by the sequential recording, because previous studies show that similar conclusions are

194 obtained by simultaneously recorded neurons and sequentially recorded neurons (Aggelopoulos

195 et al. 2005; Anderson et al. 2007; Baeg et al. 2003; Gochin et al. 1994; Nikolić et al. 2006;

196 Panzeri et al. 2003).

197 Visualization of population responses in 3D space could help us gain an intuitive

198 understanding about highly complicated neural responses (Bartho et al. 2009; Saha et al. 2013; 
199 Stopfer et al. 2003). Such visualization can be realized by principal component analysis (PCA).

200 PCA is a linear dimensionality reduction technique. It identifies a set of linearly uncorrelated

201 variables, called "principal components", from an original dataset composed of a large number

202 of possibly correlated variables and captures as much of the variability in the dataset as possible

203 (Jolliffe 2002). The principal components are ordered by the amount of variability that each

204 component accounts for, and the first principal component has the largest variance. With regard

205 to neural population responses, each single unit counts as one dimension in the population

206 response space. Given the neural response of $n$ single units in a neural population, an n-

207 dimensional response vector $R^{n}$ can be generated. By applying PCA on the n-dimensional

208 response space, we can obtain $\mathrm{m}$ virtual neurons to constitute an $\mathrm{m}$-dimensional response space

209 preserving as much of the variance in the original dataset as possible, where $\mathrm{m}<=\mathrm{n}$. If we keep

210 only the first three virtual neurons' responses $(\mathrm{m}=3)$, we can visualize the population responses

211 as a trajectory in 3D space by connecting the responses at a series of time points. In the

212 following analysis, PCA was implemented for each vocalization type. The first three principal

213 components accounted for about $30 \%$ of the original dataset's variance for each vocalization at

214 multiple intensities, and for about $22 \%$ of the variance for each vocalization at multiple SNR

215 levels, under either WGN or Babble noise. For visualization, trajectories were smoothed with a

216 10-point running window for pure vocalizations, and a 20-point running widow for noisy

217 vocalizations.

218 Based upon the trajectory visualization analysis, we can further quantify the structure of

219 trial-averaged population responses. To quantify the rotation of the population response vectors

220 in response to a particular stimulus $s_{0}$ in 3D space, the angle between a response vector $\vec{r}_{t}$ at

221 time $t$ and a reference response vector $\vec{r}_{0}$ at time $t_{0}$ can be computed as in (2) (Bartho et al. 
222 2009). By defining the first point of the spontaneous population response corresponding to

223 stimulus $s_{0}$ as the reference vector, the angle evolution of population response vectors can be

224 obtained by concatenating the angles calculated at all the available time points, $t=t_{0}, t_{1}, \ldots, t_{n}$,

225 during pre-stimulus, stimulus, and post-stimulus. We calculated the angle evolution for the five

226 vocalizations at four intensities and ten SNR levels under WGN and Babble noise conditions as

227 follows:

$$
\theta\left(s_{0}, t ; s_{0}, t_{0}\right)=\cos ^{-1}\left(\frac{\vec{r}_{t} \cdot \vec{r}_{0}}{\left|\vec{r}_{t}\right|\left|\vec{r}_{0}\right|}\right)
$$

229 corresponding to stimulus $s$ relative to response vector $\vec{r}_{s_{0}}$, which in turn corresponds to stimulus

$230 s_{0}$ across time, $t=t_{0}, t_{1}, \ldots, t_{n}$, as displayed in (3).

$$
\theta\left(s, t ; s_{0}, t\right)=\cos ^{-1}\left(\frac{\vec{r}_{s} \cdot \vec{r}_{s_{0}}}{\left|\vec{r}_{s}\right|\left|\vec{r}_{s_{0}}\right|}\right)
$$

The angles of population responses to vocalizations at three softer (15 dB SPL, $35 \mathrm{~dB}$

232 SPL, and $55 \mathrm{~dB}$ SPL) levels relative to the population response to vocalization at $75 \mathrm{~dB}$ SPL

233 were calculated. The noise effects on population response angle evolution were also investigated

234 by computing the angles of responses to noisy vocalizations and pure noise relative to pure 235 vocalizations.

236 To investigate the discriminability of population responses trial-by-trial (Bartho et al.

237 2009; Meyers et al. 2008), template-based stimulus identity predictive models were built based

238 upon neural population responses. For predictive model decoding of vocalizations at multiple 239 intensities, there are three types of models: single-bin based, sliding-bin based, and varying-cell- 
number based. To build a single-bin based model, for each stimulus condition (five vocalizations

241 at four intensities), five trials of single-unit responses at a particular time bin were randomly

242 sampled out of, at most, 10 trials for each neuron $(\mathrm{N}=326$, each has $5 \sim 10$ trials $)$. The five trials

243 were concatenated to form a $100 \times 326$ population response matrix. The stimulus identity

244 corresponding to each response trial is called a label. There are a total of five labels, each

245 representing a vocalization type $(c=5)$. Four trials of the neural population's response to each of

246 five vocalizations delivered at the highest intensity, $75 \mathrm{~dB}$ SPL, were further randomly selected

247 trials as the training templates. The remaining 80 trials of neural population responses were used

248 as the testing dataset, and the label corresponding to each trial was decoded by calculating the

249 cosine distance between this trial and the 20 template trials. The vocalization type or stimulus

250 label corresponding to the template trial with the shortest distance from the test trials was

251 assigned as the predicted label. This whole process was repeated 50 times. The performance of

252 the predictive model was evaluated by its overall accuracy and confusion matrix. The overall

253 accuracy computed the percentage of stimulus labels correctly predicted, and the confusion

254 matrix revealed more information about the chance of a particular label being predicted as one of

255 the five labels.

256 While a single-bin based model was built to investigate the neural discriminative

257 performance at each time point, a sliding-bin based model was used to study the effect of time

258 accumulation on neural discriminability. The process of building a sliding-bin based model was

259 very similar to that of a single-bin based model, except for that the response bin from each single

260 unit were varied from 1 to 41 , where 41 is the number of bins that the shortest vocalization

261 Tsikstring has. Performance as a function of temporal resolutions (time bin width) was

262 investigated with predictive model using 41 bins, and the temporal resolution was varied from 5 
$263 \mathrm{~ms}, 10 \mathrm{~ms}, 20 \mathrm{~ms}, \ldots$, to $100 \mathrm{~ms}$. Last, a varying-cell-number based model was created by

264 changing the number of neurons in the population, and only models with 41 bins were studied.

265 The population neural discriminability of vocalizations at multiple SNR levels was

266 investigated, much like that of vocalizations at multiple intensities. Predictive models under

267 WGN and Babble conditions were built separately. Here, the training templates had six labels,

268 including five pure vocalizations and one type of noise $(c=6)$. In addition, to further study

269 population decoding with a subpopulation of neurons, a predictive model for each vocalization

270 type was built by using the number of time bins available for that particular vocalization. For

271 each vocalization, a different subpopulation of neurons was included because of the contextual

272 dependent effect ( $\mathrm{Ni}$ et al. 2016). There were only two labels, vocalization and pure noise $(\mathrm{c}=2)$.

273 Population neural responses from ten SNR levels were decoded as either vocalization-present or

274 vocalization-absent. A linear support vector machine (SVM) classifier was used instead of the

275 template-based predictive model to accomplish the binary classification task. The SVM

276 classified the neuronal responses by training a separating hyperplane based upon the labeled

277 training trials and had very good performance for binary classification (Van Gestel et al. 2002),

278 while the template-based method did not have a particular training session. The generalizability

279 of the classifier over lower SNR levels was studied by using different training data, for instance,

280 neural responses to vocalizations at $20 \mathrm{~dB}$ SNR.

281 Normality was verified by the Lilliefors test. Unless otherwise indicated, hypothesis

282 testing was conducted using a two-sided Wilcoxon signed-rank test. The significance criterion 283 was set to 0.05 . 


\section{Results}

285 In this study we investigated the alternation of neural population activities evoked by two

286 types of noise, WGN and Babble, in response to marmoset conspecific vocalizations. We

287 recorded the responses of 326 marmoset A1 single-units to five vocalizations degraded by WGN

288 and marmoset Babble, and units were selected for further analysis according to the response

289 criterion for each vocalization. Power spectra of vocalizations and noises are shown in Figure

290 1A. Spectrograms of vocalization Trillphee at all the tested SNR levels under two noise

291 conditions we studied are also displayed in Figure 1B. For each vocalization, we only included

292 responsive neurons to that particular vocalization in the analysis. To measure the degree of

293 degradation that noise contributed to the neural representation of vocalizations, we calculated the

294 amount of vocalization encoded by each unit as a function of SNR. 
A

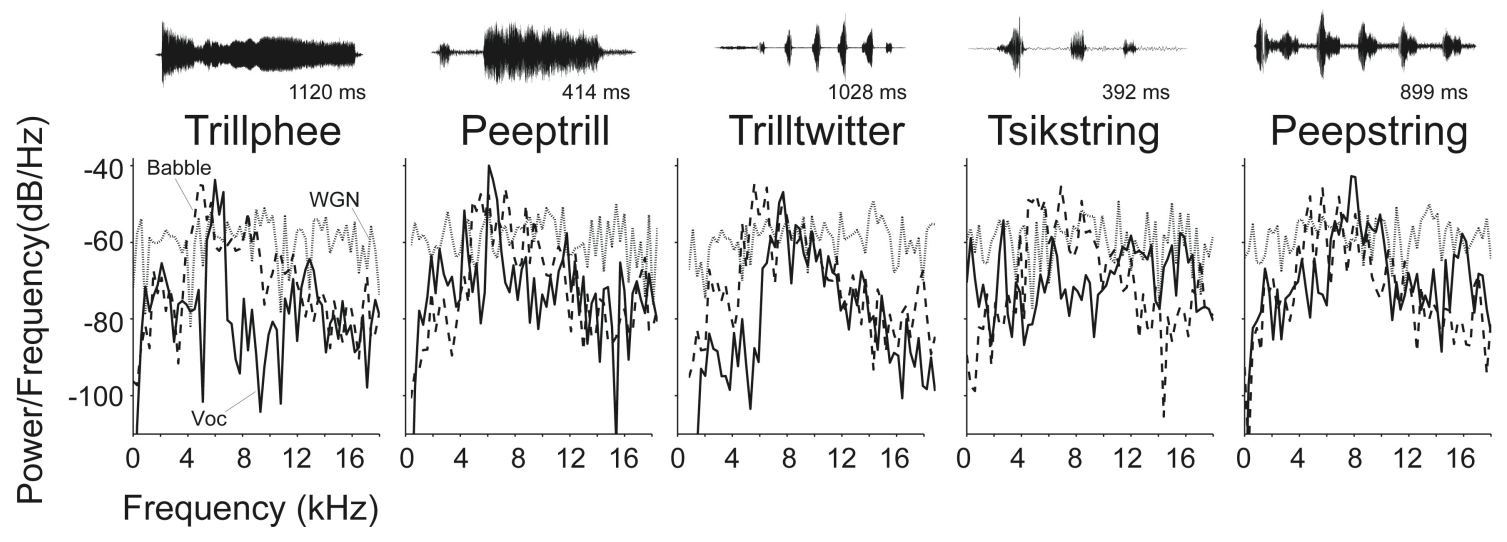

B

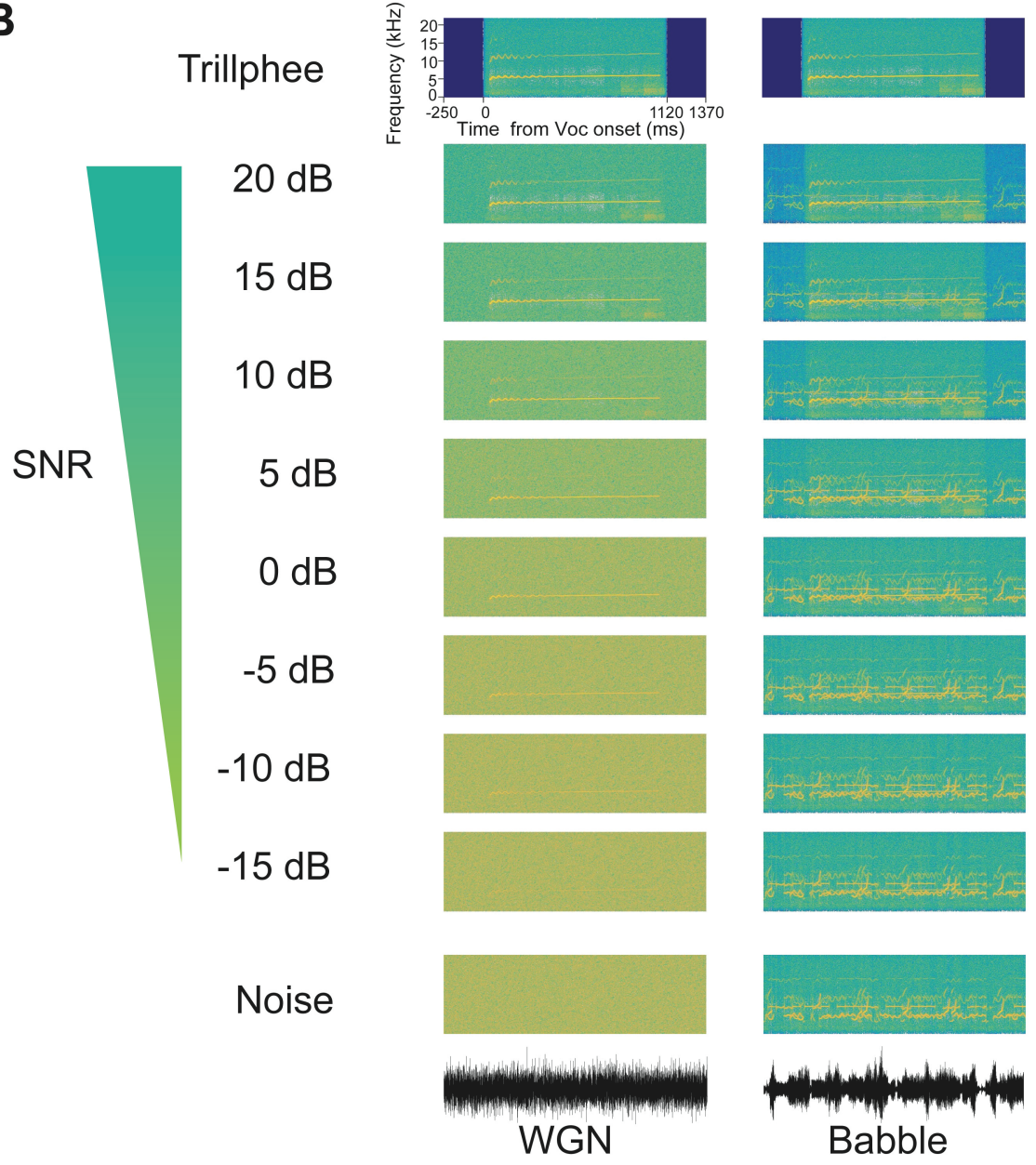

Figure 1: Acoustic stimuli used to investigate robust sound encoding in auditory cortex (A) Power spectrum of five vocalizations (solid lines), WGN (gray lines) and Babble noise (dashed lines). Background noises were truncated to have the same duration as each vocalization. The temporal waveform of each vocalization is displayed above each power spectrum. (B) Example spectrogram of vocalization Trillphee in noise at 10 different SNR levels, including pure noise and pure vocalization. The first column is Trillphee with WGN as background noise, and the second column is with Babble as background noise. The temporal waveform of WGN and Babble are shown below each column. 


\section{Population coding trajectory for clean vocalizations in $3 D$ response space}

An intuitive understanding of population neural responses can be obtained by visualizing their spatiotemporal structure. A powerful tool to achieve this is to project the high-dimensional response vectors onto a lower dimensional space, in which enough variance in the highdimensional dataset is captured by three principal components (i.e., virtual neurons).

311 were formed by connecting the response points from three time stages: pre-stimulus, during-

312 stimulus, and post-stimulus. Skeletons, which link the first point on the trajectory with the

313 remaining points, were plotted to visualize the response hyperplane. Hyperplanes belonging to

314 different vocalizations all have very distinct shapes. Some are relatively smooth and simple, such

315 as Trillphee, while some are more tangled and twisted, such as Peepstring.
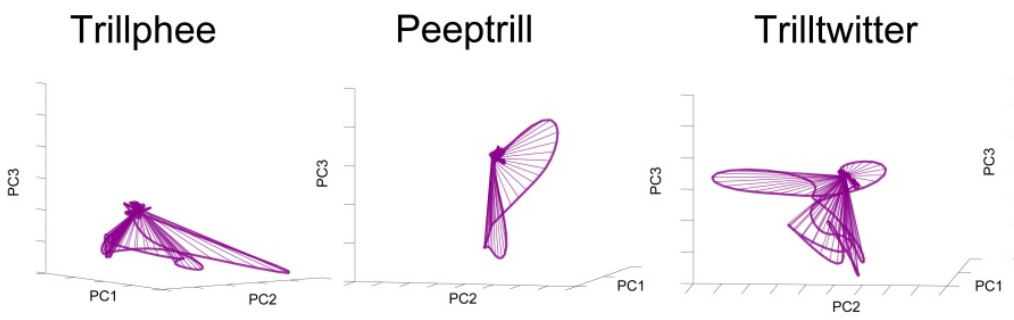

Tsikstring

Peepstring
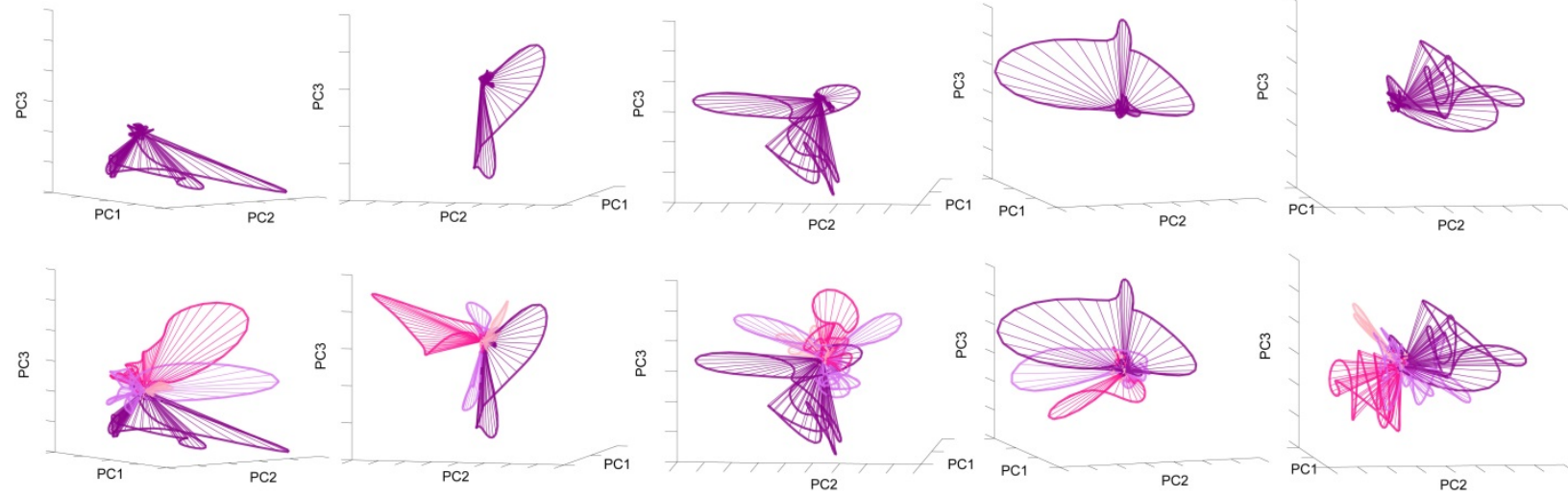


\section{Intra-trajectory angle evolution encodes stimulus identity}

How does the population hyperplane change in response to a decrease in intensity? Here

321 we consider the hyperplane at $75 \mathrm{~dB}$ SPL as the reference hyperplane. If neuronal populations

322 linearly scaled their responses' amplitudes, we would expect to see the response hyperplane

323 shrink without changing its position in 3D space. Alternatively, the hyperplane could change in a

324 way that only rotates its position relative to the reference hyperplane. As a matter of fact, the

325 hyperplane seems to both resize and rotate. It is worth noting that the more the intensity

326 decreases, the further the hyperplane deviates from the reference, in a consistent direction.

327 To quantify the response hyperplane and the changed induced by intensity, we calculated

328 the angle between response vectors in two ways. First, we quantitatively described the

329 spatiotemporal structure of a hyperplane by computing the angle between the first response

330 vector on the hyperplane and the remaining response vectors over time in Figure 3. Clearly,

331 across vocalizations and intensities (except for $15 \mathrm{~dB}$ SPL), the angle fluctuated between 0 and

33260 degrees at the pre-stimulus stage. To process the upcoming stimulus, an acute increase in the

333 angle immediately followed the stimulus onset and further evolved during the stimulus

334 presentation. As the end of the stimulus presentations approached, the angles acutely declined

335 back to the pre-stimulus level. Therefore, the angles of response vectors during the stimulus

336 presentations occupied a distinctively different range from the pre/post presentation. Comparing

337 the angles over time across different intensities, we noticed that at measured intensities above 15

$338 \mathrm{~dB}$ SPL, the angles over time were very similar without the scaling shown in the spiking rate and

339 response variability. This similarity indicates that population responses may represent a

340 vocalization identity in an intensity-invariant manner by encoding the information in the angle

341 evolution of a trajectory. 


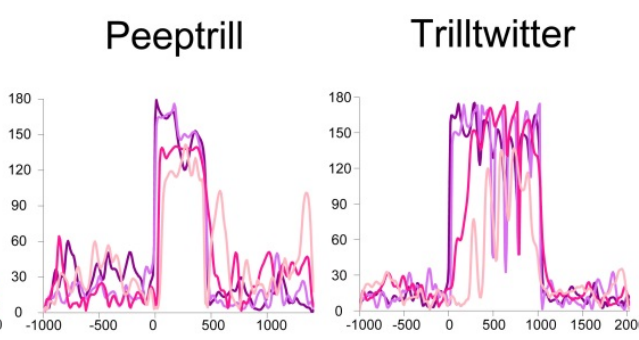

Trilltwitter Tsikstring
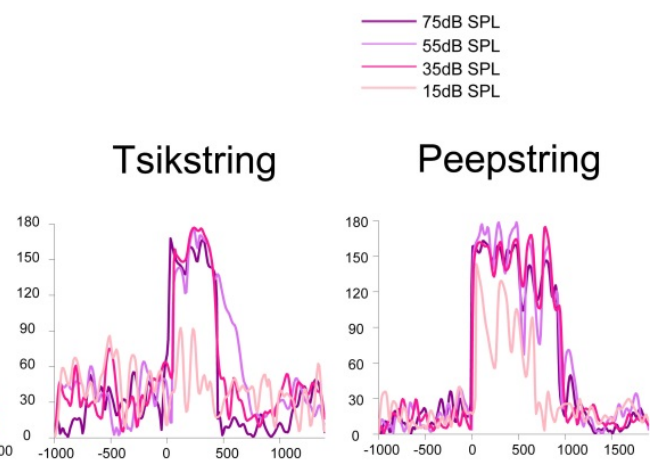

Figure 3: Evolution of rotation angles relative to the first time point (in silence) of the population response at multiple intensities in $3 \mathrm{D}$ space

Inter-trajectory angle evolution encodes stimulus intensity

Next, we quantified the influence of intensity on the deviation of response trajectories by computing the angles between response trajectories of decreasing intensities relative to the reference trajectory at $75 \mathrm{~dB}$ SPL over time, as shown in Figure 4. The less intense the vocalization, the further away the corresponding population trajectory was from the reference trajectory in terms of angle rotations, which is consistent with a qualitative visual inspection in Figure 2. The rotation angles, however, are not equal over time. The pre-stimulus and poststimulus periods have rotation angles that fluctuated in the same range as that in Figure 3 . For the stimulus-driven angle evolution, finer structures potentially related to the acoustic features of vocalizations can be observed. With regard to the angles between two neighboring intensities, for instance, $55 \mathrm{~dB}$ SPL vs $35 \mathrm{~dB}$ SPL, their difference at each time point is generally smaller than

357 the angle difference between different points belonging to the same intensity. Rotation of 358 population responses may serve as an indicator to encode the information of intensity.

360 structures within trajectories in 3D space at multiple intensities. By contrast, the relationship between hyperplanes at different intensities is more complicated than just an equal angle shift. 

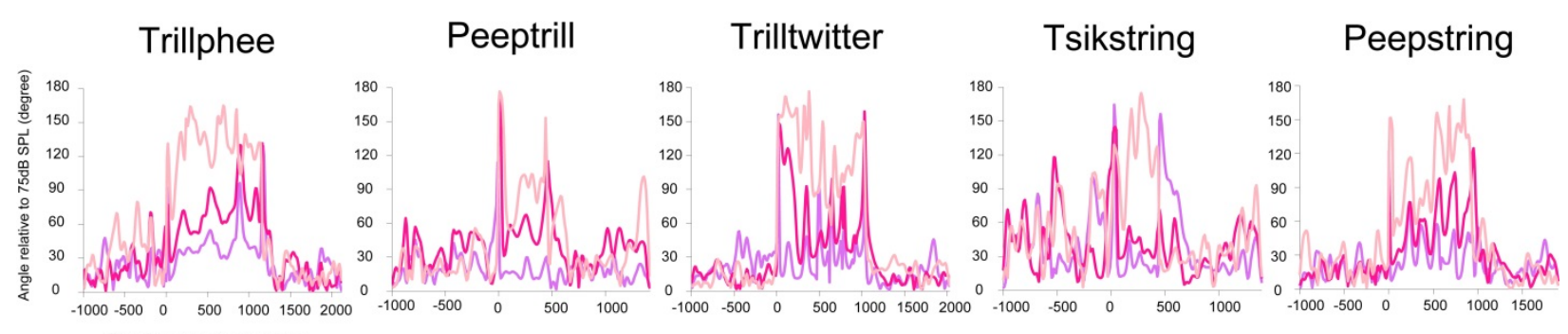

Figure 4: Evolution of the rotation angles of population responses at multiple intensities relative to the population response at $75 \mathrm{~dB}$ SPL in $3 \mathrm{D}$ space

Visualization of population coding trajectory for noisy vocalizations in $3 D$ response space

To characterize the spatiotemporal structures of population responses to vocalizations

369 with increasing amounts of noise, the associated population response trajectories based upon

370 three principal components are displayed in Figure 5. Responses were projected to different 3D

371 spaces under two noise conditions, but a salient differences can detected between how increasing

372 the amount of different types of noise affects the population response structures. Under the WGN

373 condition, two groups can be identified. Trajectories to vocalization and $20 \mathrm{~dB}$ SNR are clustered

374 together, while trajectories to $-10 \mathrm{~dB}$ SNR and pure WGN noise share a similar subspace. In

375 contrast, trajectories to vocalizations masked with Babble noise don not form individual clusters,

376 with a large portion overlapped across SNR levels. 


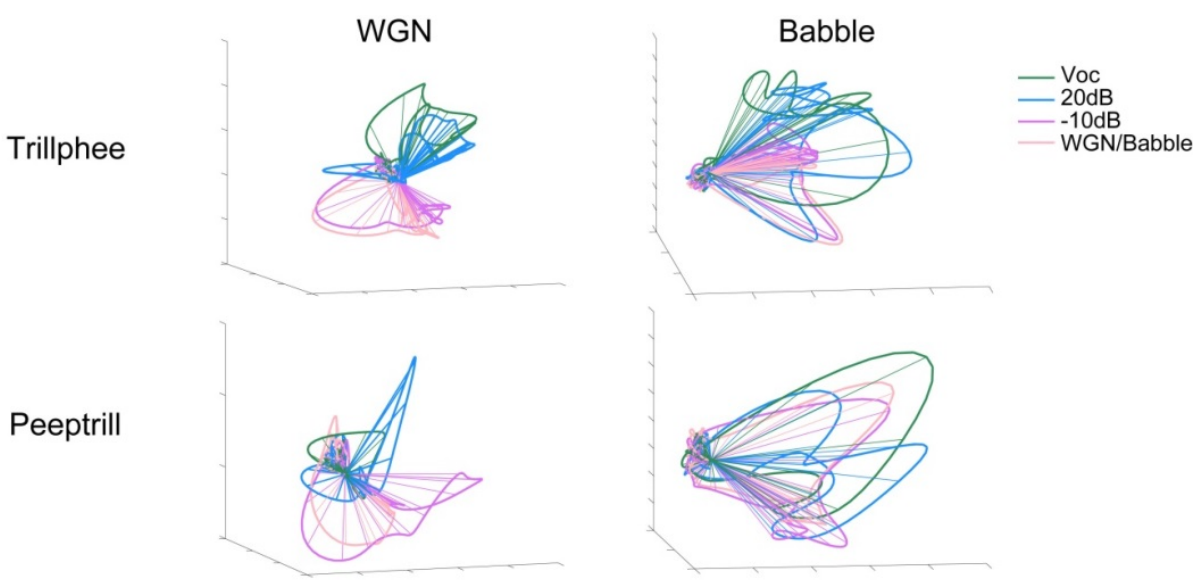

Trilltwitter
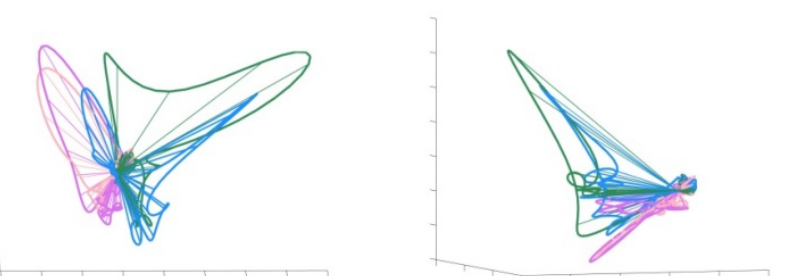

sikstring
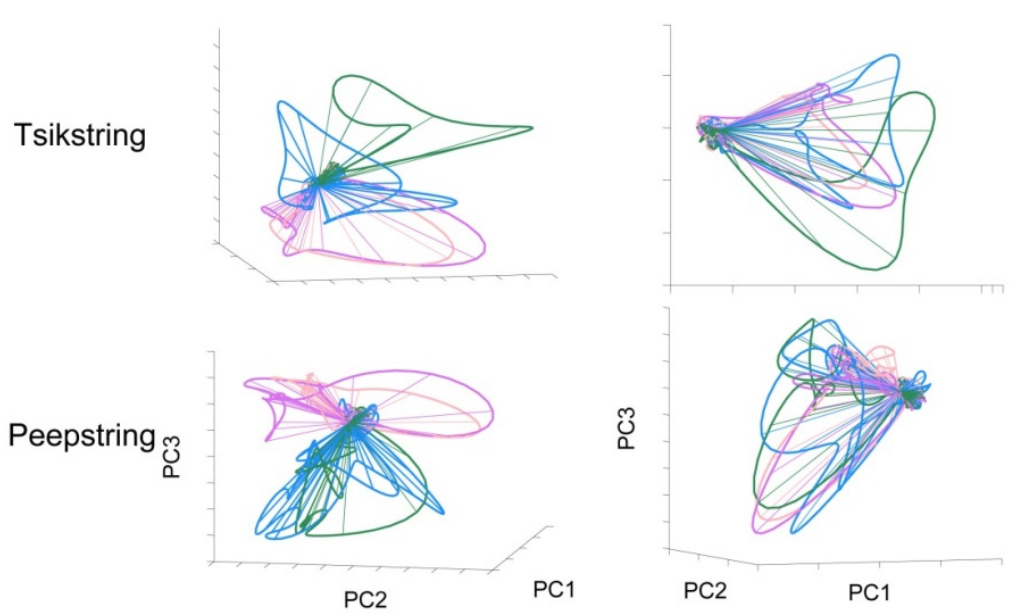

378 Figure 5: Population-averaged responses to five vocalizations in noise at multiple intensities 379 in 3D response space

\section{Inter-trajectory angle evolution encodes stimulus SNR}

Rotation angles of each trajectory are quantified in Figure 6. Trajectories start with a pre-

383 stimulus portion fluctuating below 60 degrees, greatly increase rotation angles to over 150

384 degrees following the stimulus onset, and further evolve during stimulus presentation, with

385 particular structures associated with each vocalization. Trajectories at $20 \mathrm{~dB}$ SNR share a 
386 majority of features with those of clean vocalizations, and trajectories at -10 dB SNR are more

387 noise-like. Again, angle evolution within trajectories of pure vocalizations and pure noise are

388 more separated from each other in the WGN condition than in the Babble condition. 
bioRxiv preprint doi: https://doi.org/10.1101/2019.12.21.886101: this version posted December 23, 2019. The copyright holder for this preprint (which was not certified by peer review) is the author/funder, who has granted bioRxiv a license to display the preprint in perpetuity. It is made available under aCC-BY-NC-ND 4.0 International license.
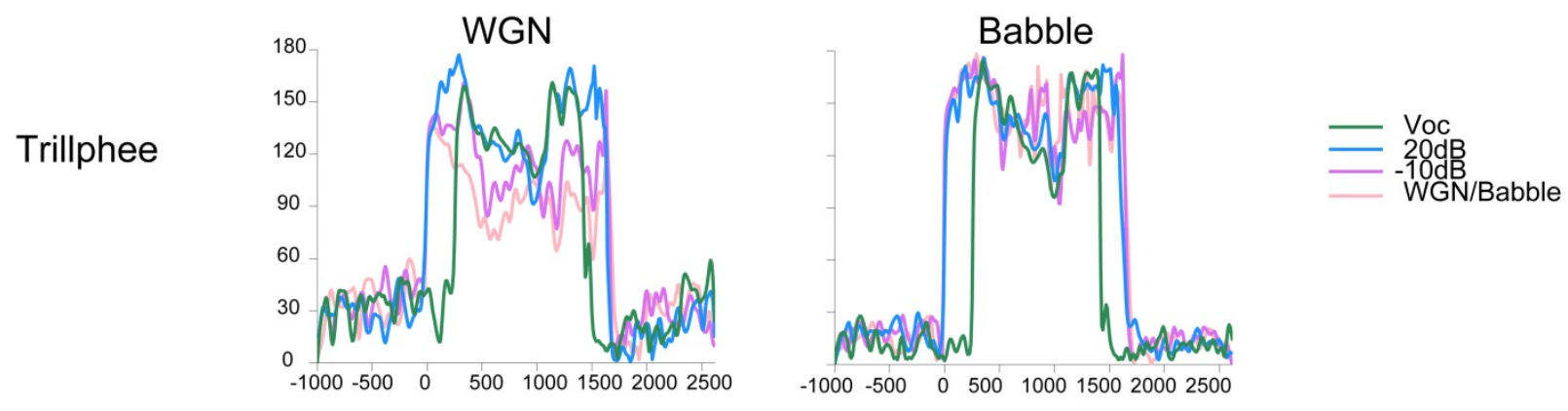

rilltwitter
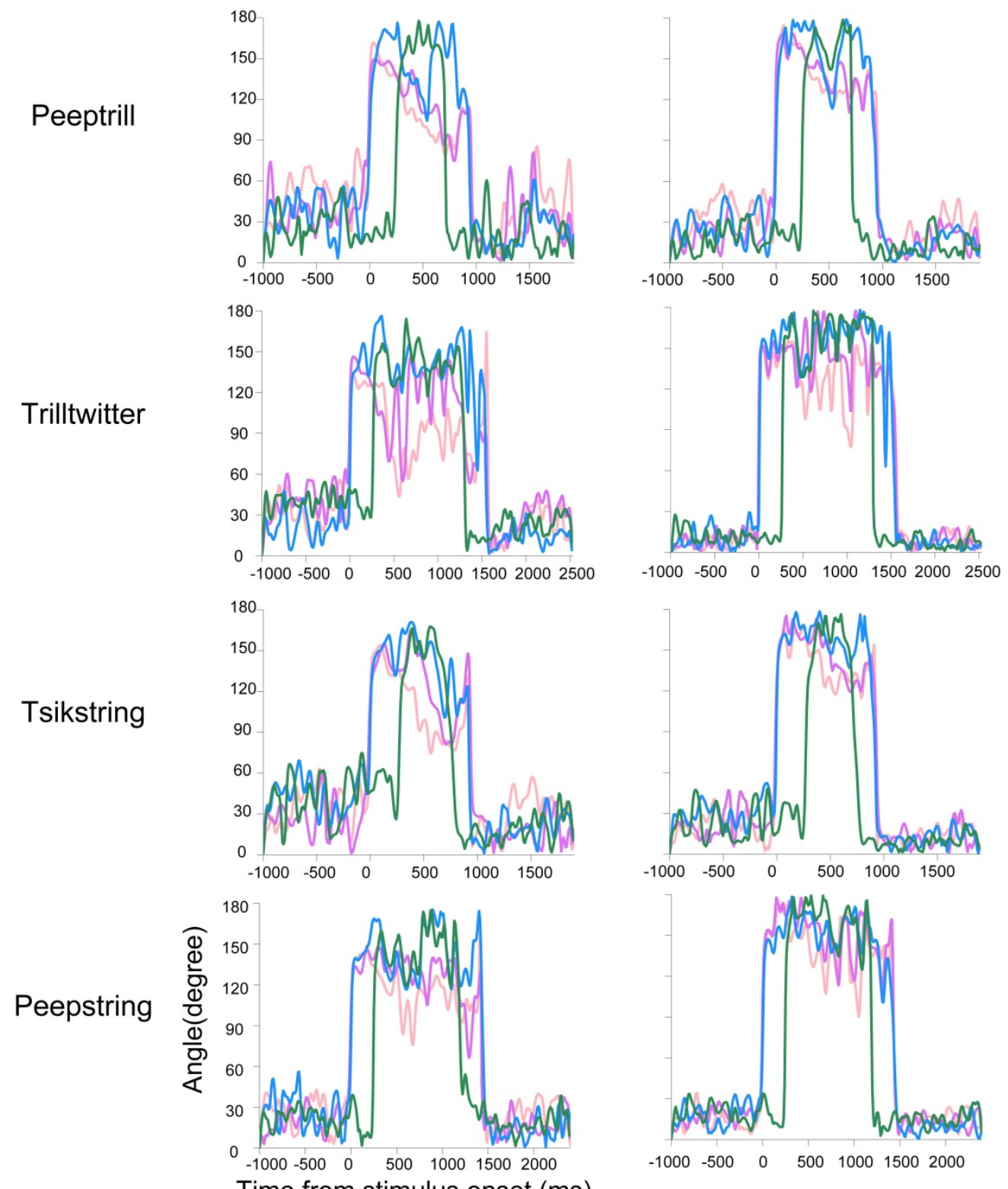
To quantify the distance between trajectories, we computed the rotation angles of

393 trajectories of vocalizations at multiple SNRs levels relative to the trajectories of pure

394 vocalizations, with the results shown in Figure 7. Two big peaks indicate the onset and offset

395 responses induced by the two 250-ms noise segments. Time courses between these two peaks

396 show that trajectories at $20 \mathrm{~dB}$ SNR have the smallest angular difference from that of pure

397 vocalizations, below 30 degrees. Trajectories of -10 dB SNR and pure noise are further away.

398 Figure 5.11 also quantitatively shows that WGN leads to more separated response trajectories

399 than Babble. 


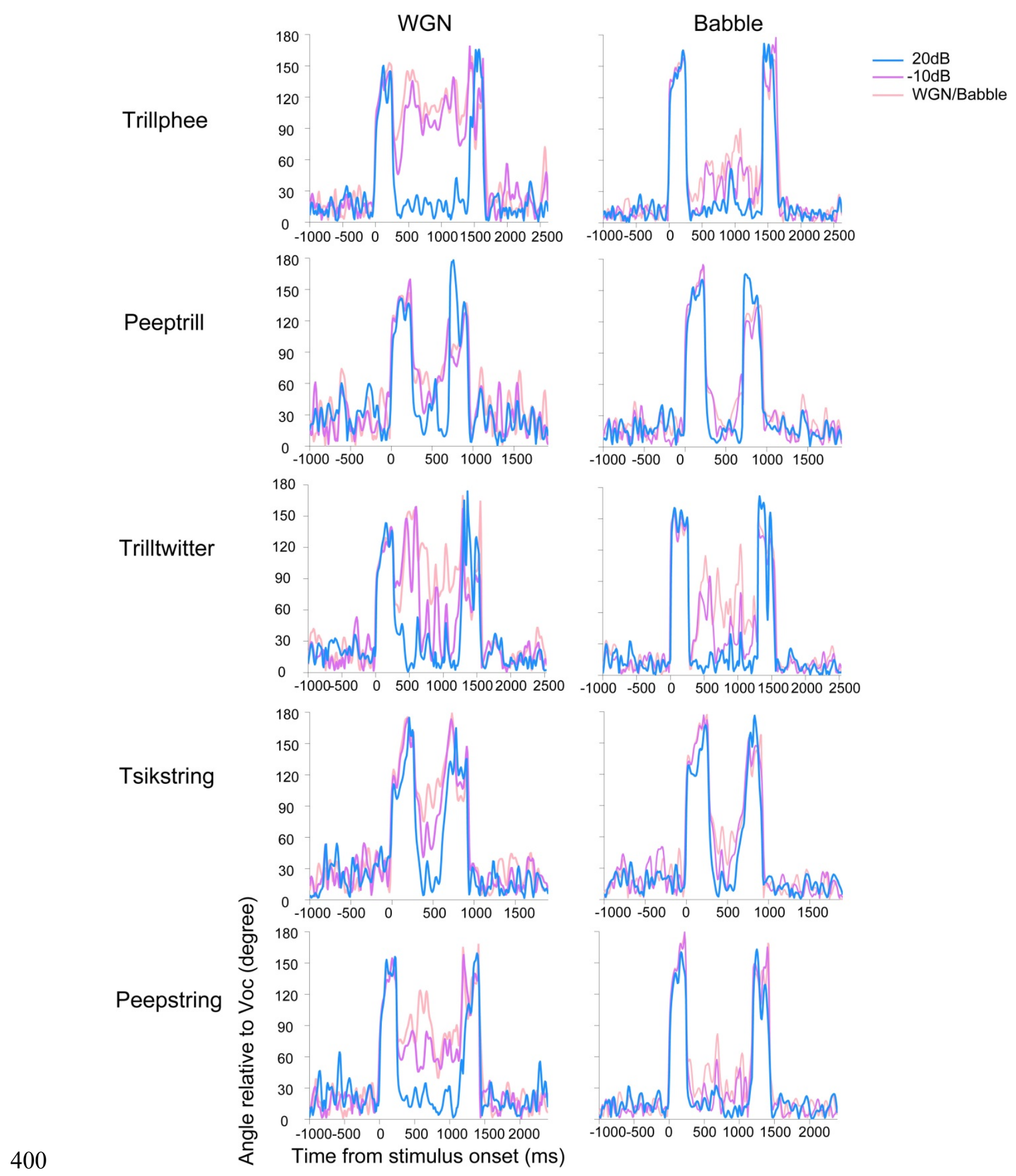


Influence of temporal resolution and temporal integration window on population neural 403 discrimination

404 In previous analyses, we studied the variability and spatiotemporal structures of 405 population responses to vocalizations at multiple intensities. Population responses averaged over

406 five to ten trials exhibited rich temporal dynamics in terms of rotation angles. Marmoset

407 vocalizations have features spanning a wide range of time scales (Agamaite et al. 2015). Here,

408 we further ask how the trial-by-trial population response discrimination between vocalizations at

409 multiple intensities depends on the temporal resolution. How does the discrimination evolve over

410 time? And how does the number of neurons in the population affect the discrimination?

411 We quantified the discriminability of population spike trains by building predictive

412 models to decode vocalization types based upon a series of different temporal resolutions, which

413 were used to bin spike trains into response vectors (spike trains of all vocalizations were

414 truncated to the length of the shortest vocalization). As shown in Figure 8, the discrimination

415 accuracy reaches an optimal level at $\sim 10 \mathrm{~ms}$, and degrades substantially with widened temporal

416 resolutions. The minimum temporal resolution we tested here is $5 \mathrm{~ms}$, and performance at that

417 level also shows a decreasing trend. 


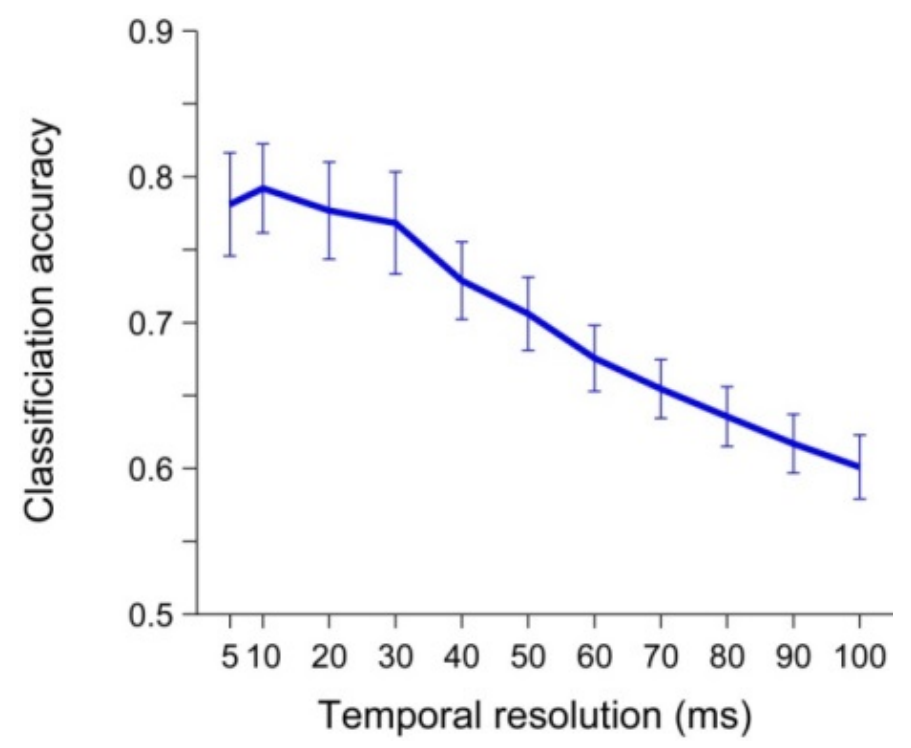

420 Figure 8: Discriminability of population spike trains in response to vocalizations at a 421 variety of temporal resolutions

424 a single time bin and with increasing numbers of time bins, and obtained the results in Figure 9.

425 Performance of predictive models based upon spontaneous activities is displayed in Figure 9A,

426 along with that of models based upon stimulus-driven activities, as a control. Discrimination

427 with a single time bin begins at the chance level, gradually increases following the onset of 428 vocalizations, and achieves a steady state within $200 \mathrm{~ms}$ after stimulus onset. Discrimination 429 with increasing lengths of spike trains is shown in Figure 9B, demonstrating a similar but 430 slightly different trend. It also begins at the chance level, and steadily increases at a relatively

431 fast speed within the first $100 \mathrm{~ms}$ after the onset of vocalizations. Later, it enters an oscillating 432 and slowly increasing mode for about $300 \mathrm{~ms}$, and finally reaches a plateau not long before the 433 whole spike train is concluded. 
A

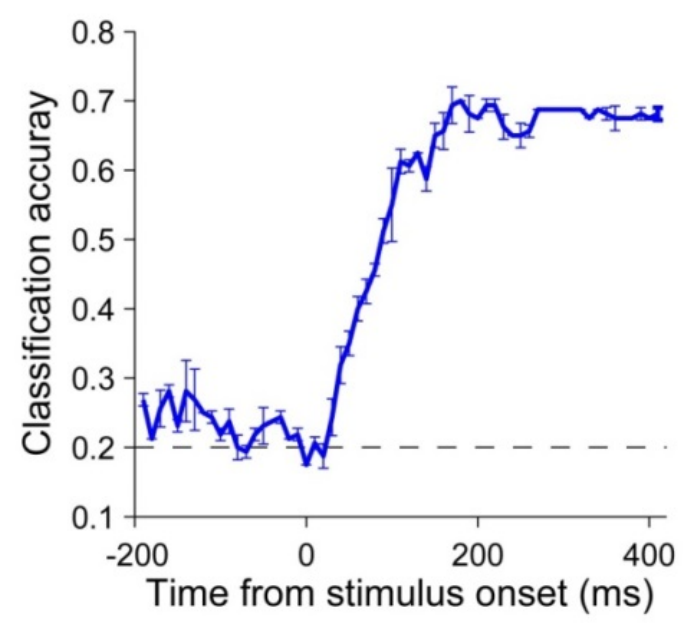

B

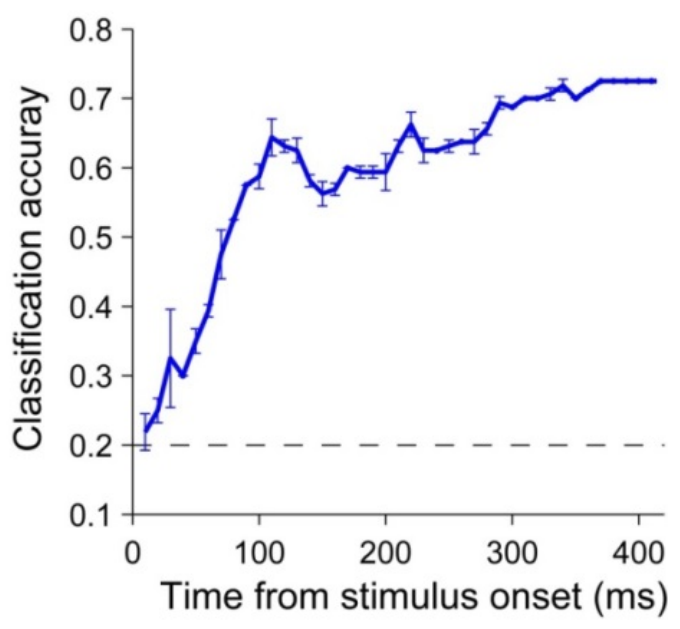

Figure 9: Dynamic discriminability of population spike trains in response to vocalizations over time

(A) Population discrimination of clean vocalizations based on single time bins evolving over time.

(B) Population discrimination of clean vocalizations based on increasing length of time bins evolving over time.

Lastly, to evaluate the influence of the number of neurons on discriminability, we randomly sampled various numbers of neurons to build predictive models classifying 20 stimulus labels, until all neurons were included. The resulting discrimination analysis for each vocalization intensity condition is displayed in Figure 10. Generally speaking, as more and more neurons are included as shown in Figure 10A, discrimination improves from the chance level to a plateau when the neuron numbers are between 200 and 300. Neural responses to all vocalizations at $75 \mathrm{~dB}$ SPL can be $100 \%$ classified when enough neurons are included. The responses at other intensities, however, are not all well classified. In addition, a relative higher intensity does not necessarily guarantee a better performance, as seen by comparing the $55 \mathrm{~dB}$ SPL performance of Trillphee and Peeptrill comparing with their 35 dB SPL performance. A better evaluation of the classification performance can be obtained by the confusion matrix in Figure 10B. The confusion matrix provides a clearer view of how likely each neural response to 
454 a particular stimulus is to be mistakenly classified as another label. It shows that neural responses

455 to the same vocalization but at different intensities above $15 \mathrm{~dB}$ SPL are less likely to be

456 classified as other vocalization types. The matrix demonstrates that the vocalization type is

457 relatively more robustly encoded than the intensity. At $15 \mathrm{~dB}$ SPL, misclassified labels seem to

458 be equally distributed among different vocalizations, which makes sense given that the

459 vocalizations are hardly audible at that level.

\section{A}
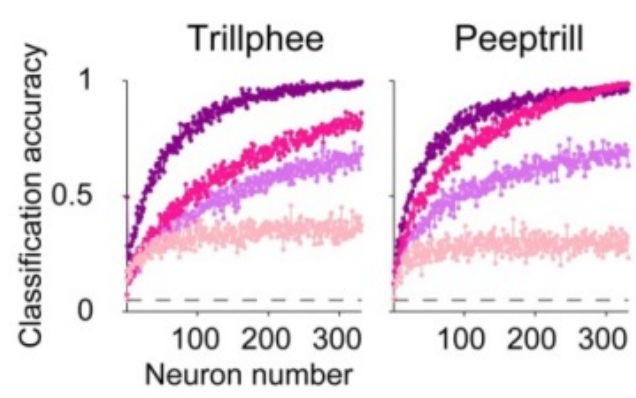

\section{Trilltwitter}
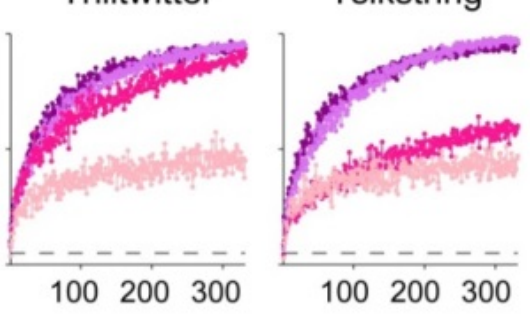

B

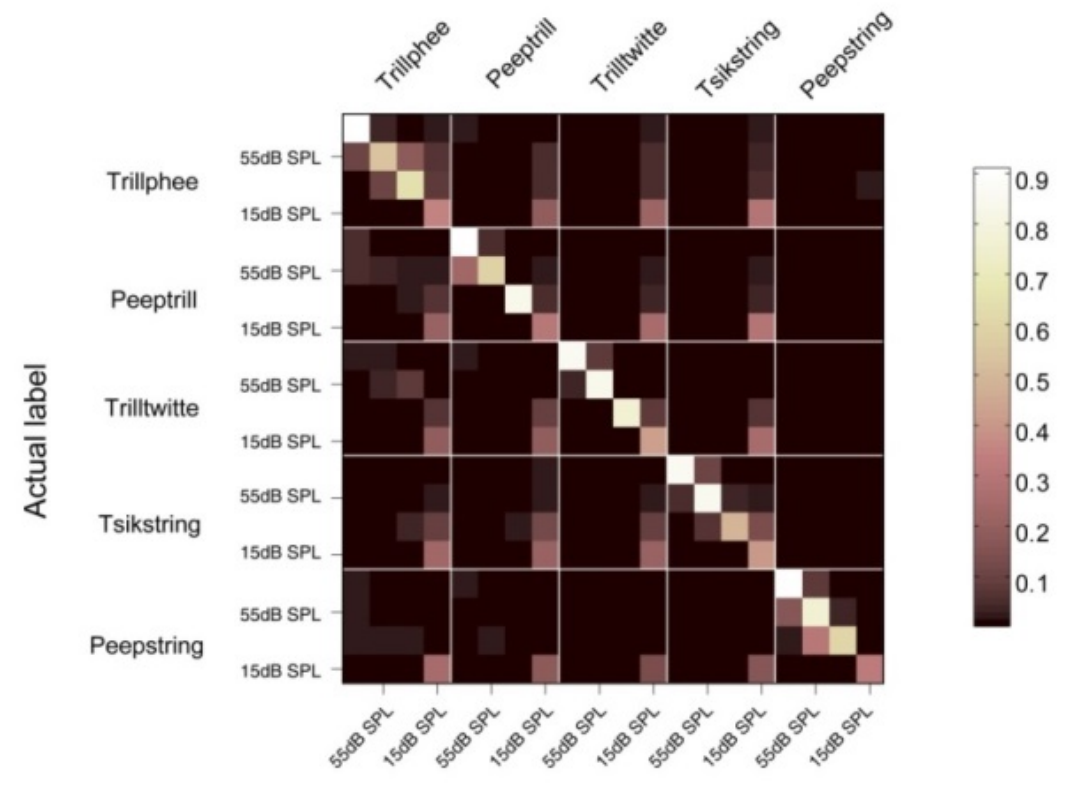

461 Figure 10: The influence of the number of neurons on population discriminability

462 (A) Classification accuracy of population responses of five clean vocalizations at different 463 intensities.

464 (B) Confusion matrix of population responses classification. 
To evaluate the dependence of discriminability of population responses for vocalizations across multiple SNRs on the temporal resolution, we built predictive models based upon spiking

467 trains binned by time windows of different lengths. Models were built to classify single trial

468 population response to one of the five vocalizations or pure noise $(c=6)$, and evaluated by the

469 percentage of correctly classified labels. Here, the number of time bins possessed by the shortest

470 vocalization was used. The temporal resolution, in Figure 11, appears to negatively associated

471 with the classification accuracy. Based upon the range of time bins we investigated $(5 \mathrm{~ms} \sim 100$

$472 \mathrm{~ms}$ ), a finer temporal resolution appears to provide a better discriminability. In addition,

473 discrimination performance under WGN is about twice that under Babble.

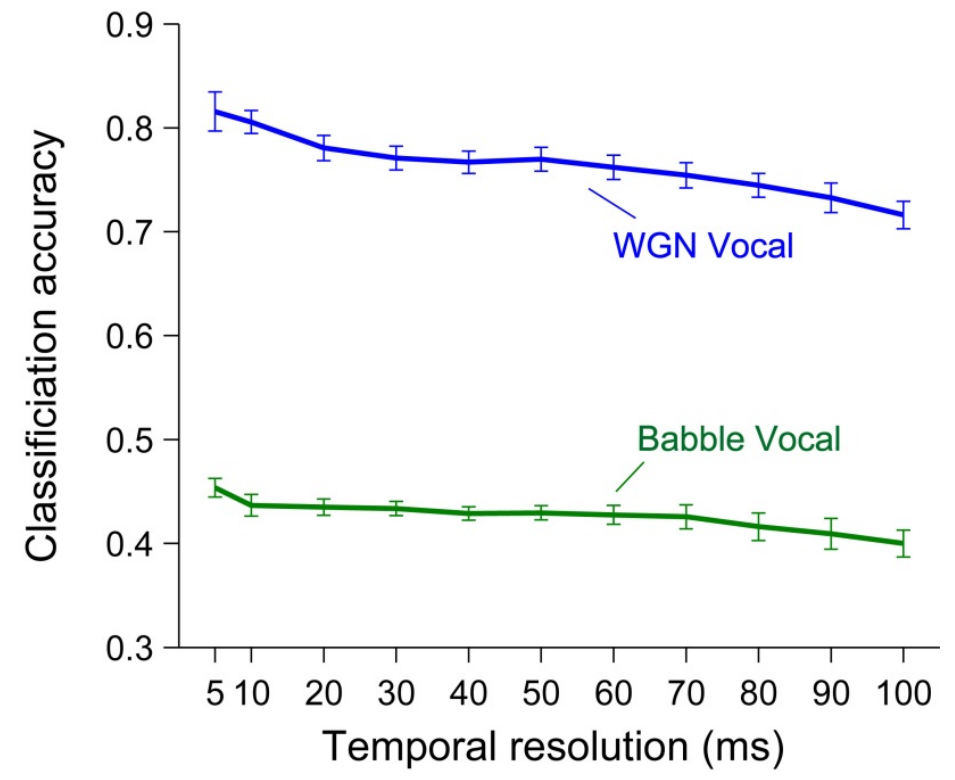

Figure 11: Discriminability of population spike trains between pure noise (WGN/Babble) and noisy vocalizations at a variety of temporal resolutions

More details of the classification performance can be obtained by segregating the accuracy for each vocalization and SNR level as in Figure 12. The performance of each vocalization is

480 displayed as a function of SNR in Figure 12A. For the WGN condition, neural responses to 481 vocalizations delivered with SNR above $5 \mathrm{~dB}$ can largely be identified as driven by the correct 
482 vocalization type, and neural responses delivered with SNR under -10 dB SNR are most likely to

483 be classified as purely noise-induced, with $-5 \mathrm{~dB}$ and $0 \mathrm{~dB}$ as the transition points. Neural

484 responses to vocalizations under Babble noise tend to have higher detection thresholds between 0

$485 \mathrm{~dB}$ SNR and $10 \mathrm{~dB}$ SNR. Under both noise conditions, Peepstring vocalization had the best

486 discrimination over lower SNR levels than other four vocalizations. Whether those wrongly

487 classified neural responses were classified as other types of vocalization of pure noise can be

488 further inferred from Figure 12B. The confusion matrices clearly show that neuron responses

489 driven by a particular vocalization are rarely wrongly classified as other types of vocalizations,

490 except for Tsikstring at $-5 \mathrm{~dB}$ under WGN condition. Noises, instead, exert more interference on

491 the neural responses. 

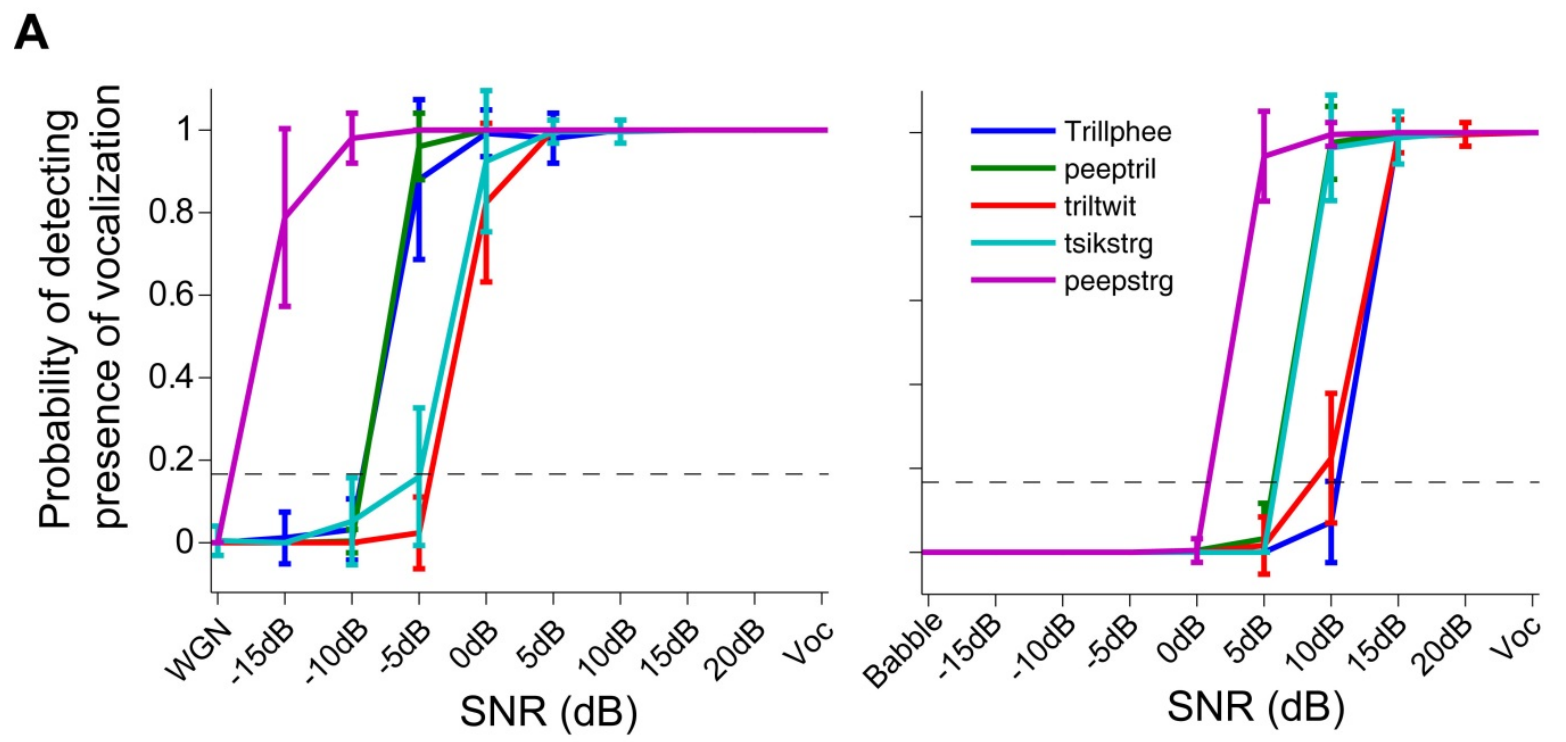

B

\section{Trillphee}
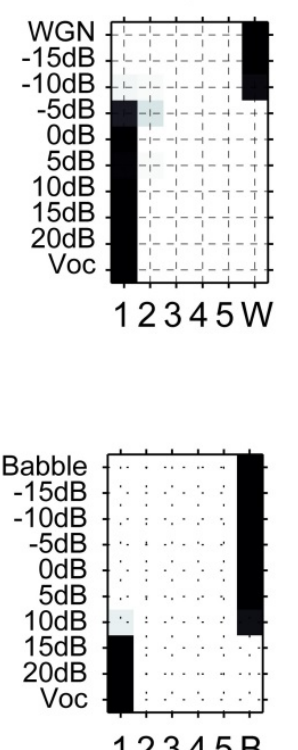

$12345 B$
2. Peeptril

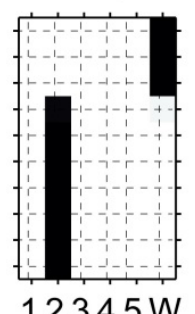

$12345 \mathrm{~W}$

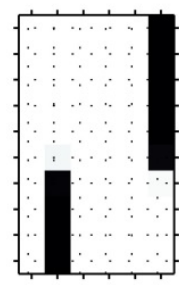

$12345 \mathrm{~B}$
3. Trilltwitter
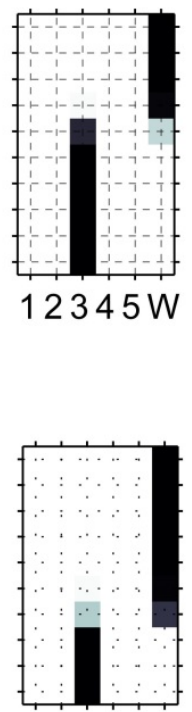

$12345 B$
4.Tsikstring
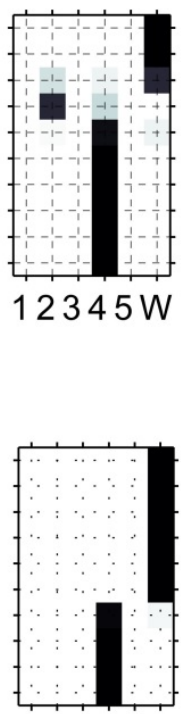

$12345 B$
5. Peepstring

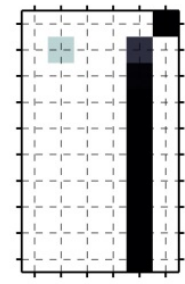

$12345 \mathrm{~W}$

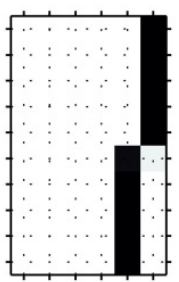

$12345 B$

\section{Predicted Label}

Figure 12: Discriminability of population spike trains between pure noise (WGN/Babble) and noisy vocalizations at a variety of temporal resolutions

495

(A) Discriminability of population spike trains between pure noise (WGN/Babble) and noisy vocalizations as a function of SNR.

497

(B) Confusion matrices of population response discriminability between pure noise (WGN/Babble) and noisy vocalizations. 
We further studied the evolution of population discrimination over time by building

501 predictive models using a single time bin and increased numbers of time bins, as shown in

502 Figure 13. Discrimination based upon a single bin begins at the chance level in Figure 13A,

503 stabilizes at 0.1 for $250 \mathrm{~ms}$ of noise preceding the vocalization, and steadily increases following

504 the onset of vocalization in the auditory scene (Figure 1). Babble has a rather low performance

505 based upon single bin response, even below the chance level as shown in Figure 13B. When

506 information was integrated over more and more time bins, the discrimination of population

507 neural responses improved with a steep slope for the first $100 \mathrm{~ms}$ following the vocalization

508 onset, and were further boosted under the WGN condition, but reached a plateau under the

509 Babble condition.

A

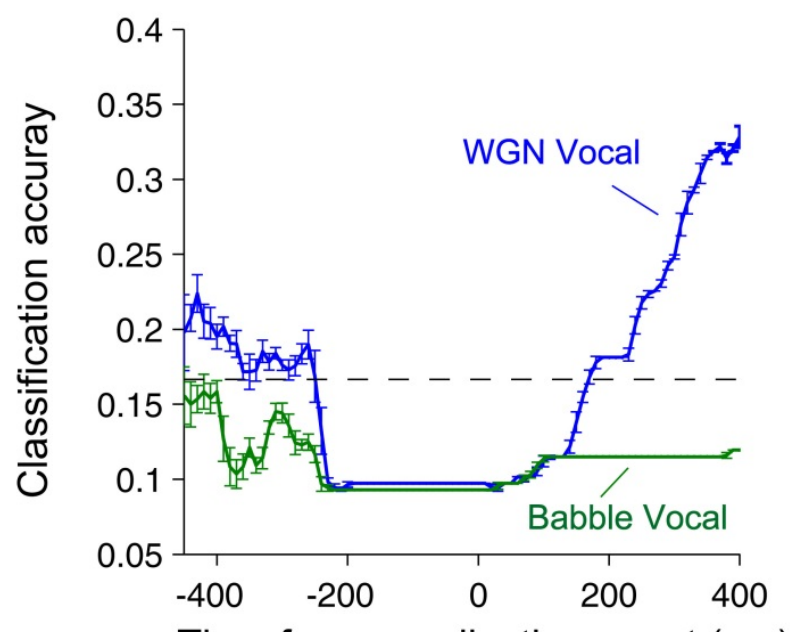

510 vocalizations over time time. evolving over time.
B

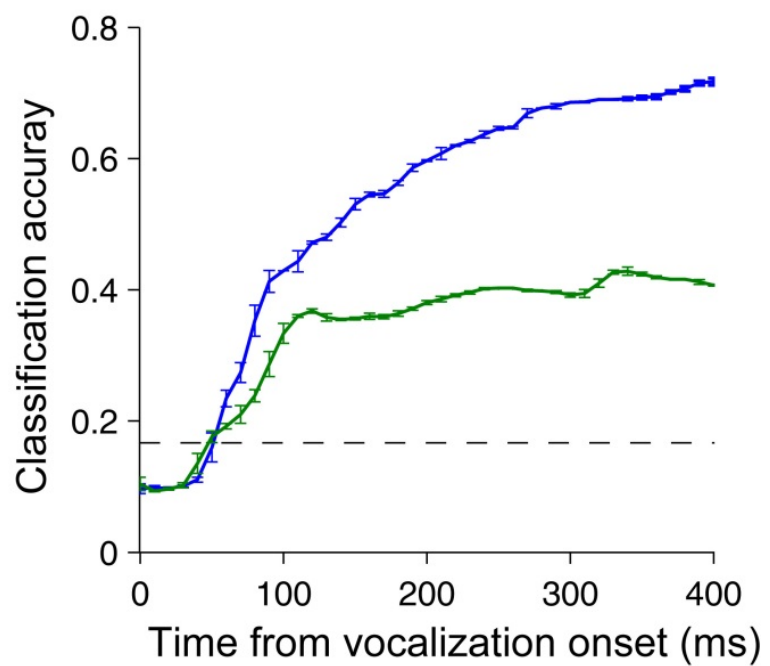

Figure 13: Dynamic discriminability of population spike trains in response to noisy

(A) Population discrimination of noisy vocalizations based on single time bins evolving over

(B) Population discrimination of noisy vocalizations based on increasing length of time bins 


\section{Optimal sub-population of neurons with the best discriminative ability}

In a previous report, we showed that responses of individual neurons to noisy

520 vocalizations can be categorized into four different groups: robust, balanced, insensitive, and

521 brittle (Ni et al. 2016). Here, we investigate the discrimination of subpopulations of neurons by

522 using pure vocalization, $20 \mathrm{~dB}$ SNR and pure noise collectively to train the classifiers. Two

523 subpopulations of neurons are shown in Figure 14: the robust group of neurons and population

524 of neurons excluding the brittle group. In Figure 14A, robust groups of neurons generally

525 produce classifiers with a slightly lower detection threshold, but their performance curves are

526 less smoothed. Considering all neurons except the brittle group in Figure 14B adds smoothness

527 and consistency between vocalizations. 

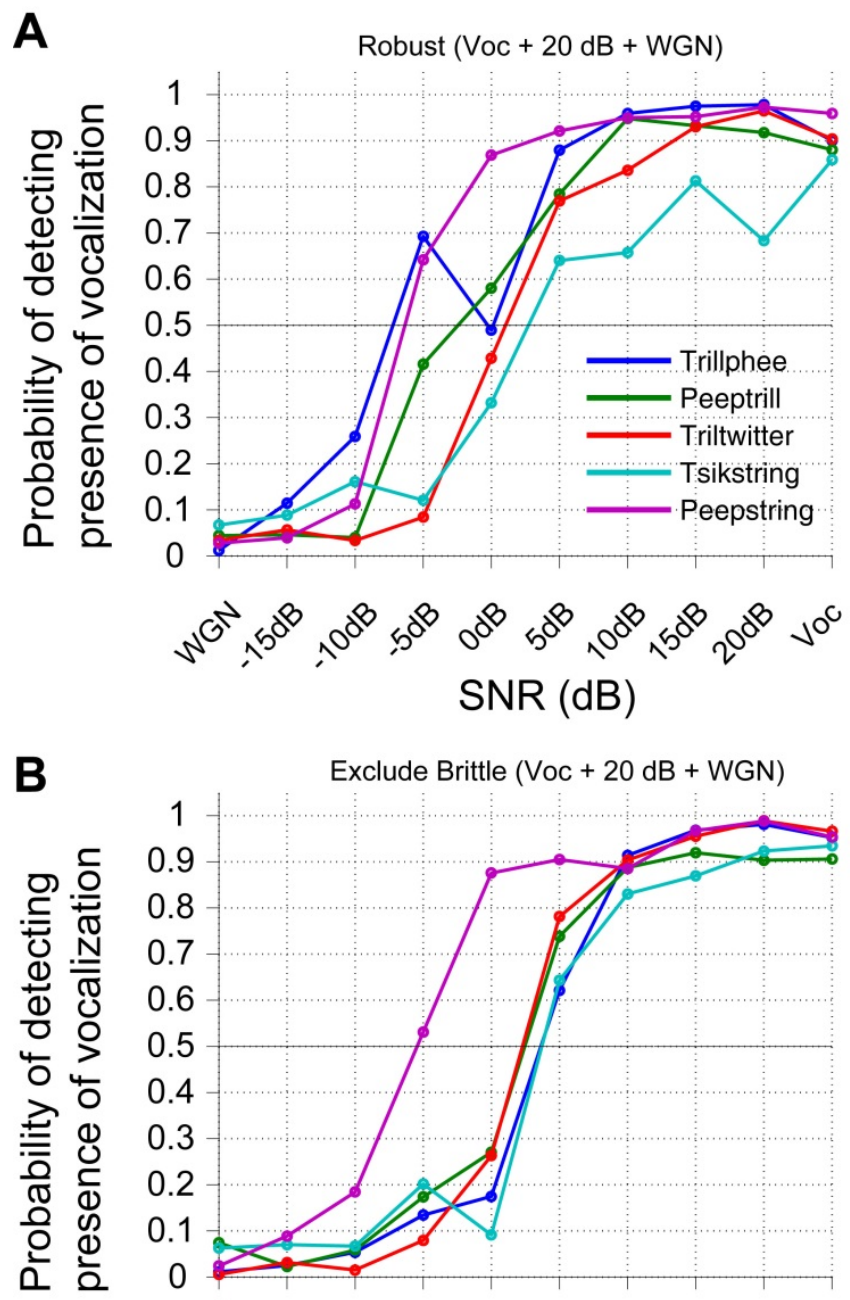

Exclude Brittle $(\mathrm{Voc}+20 \mathrm{~dB}+\mathrm{WGN})$

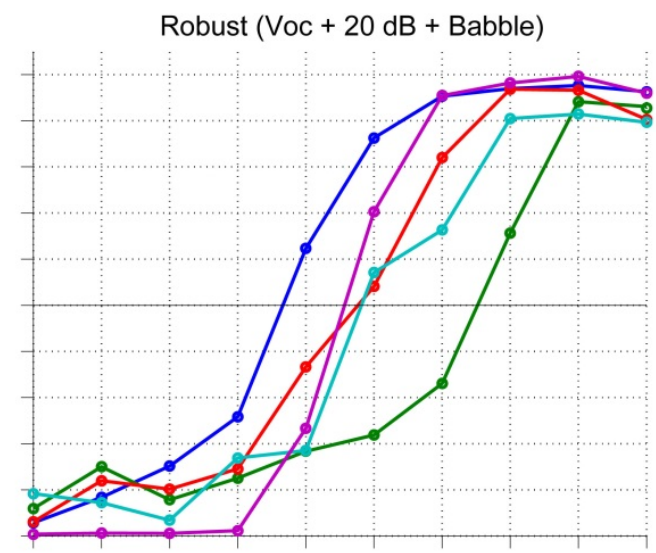

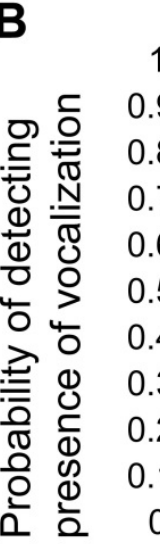
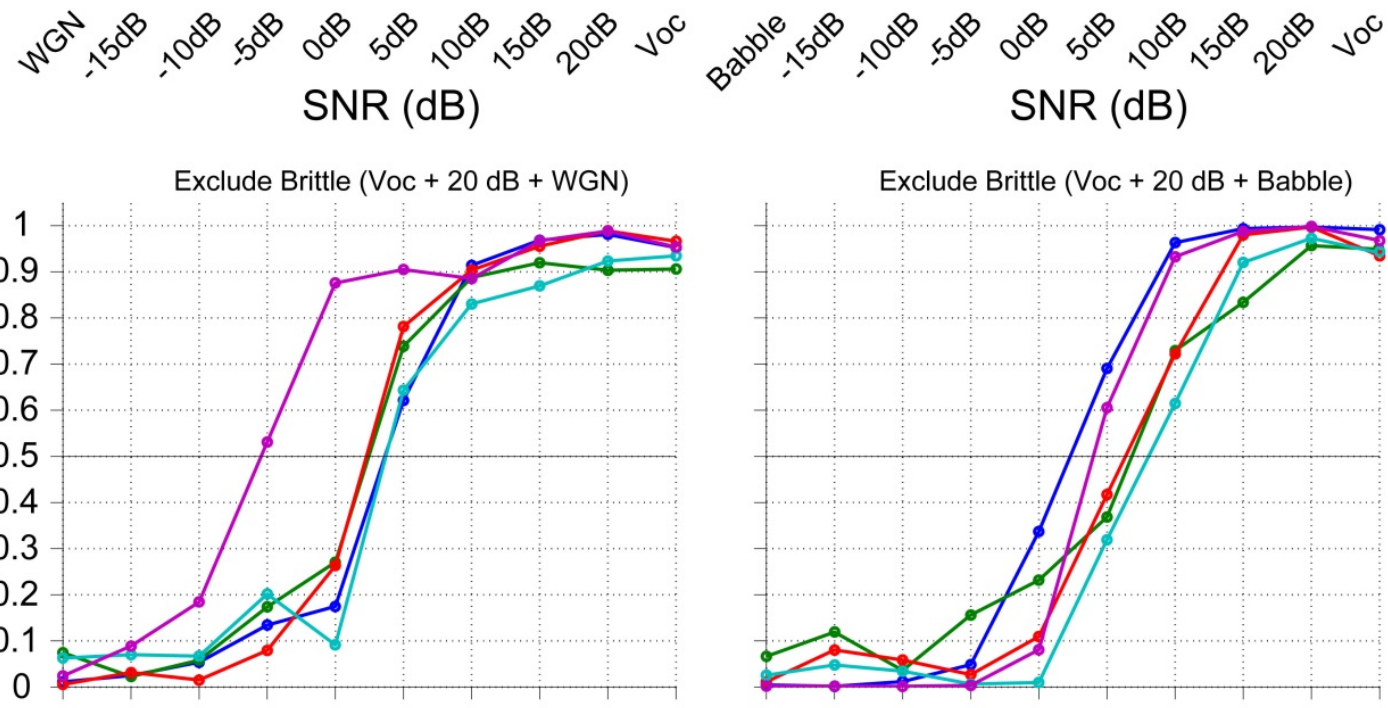

Exclude Brittle $(\mathrm{Voc}+20 \mathrm{~dB}+$ Babble $)$

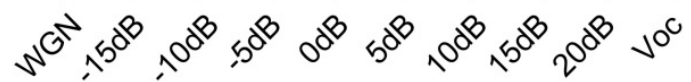

SNR (dB)

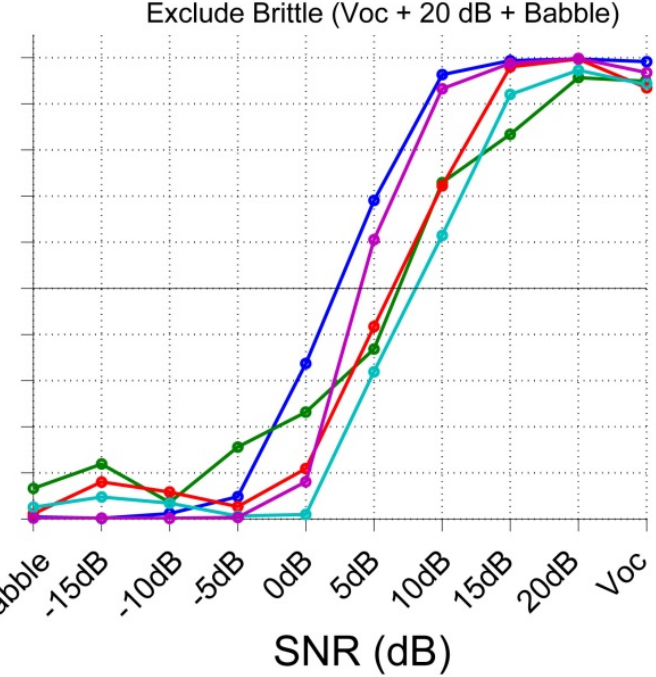

Figure 14: Optimal sub-population for discrimination

(A) Performance of subset of robust neurons discriminating noisy vocalizations.

531

(B)Performance of subset of neurons including robust, balanced, and insensitive to discriminating noisy vocalizations.

Noise enhances detection threshold

Performances of classifiers heavily depend on the quality of the training dataset. In the machine learning field, it is well known that adding an extra small amount of noise to the

536 training dataset can improve the classifiers' generalization and obtain better performance

537 (Bishop 1995). Here, we explored the generalization of neural response classifiers by using

538 different training datasets. 
For each vocalization, we built separate binary SVM classifiers by using different

540 numbers of time bins, ranging from a single time bin to all the time bins available to that

541 vocalization. Given a trial of population response, the task of the classifiers was to predict

542 whether the response was induced by pure noise or not. The performances of classifiers were

543 averaged over different numbers of time bins. Two groups of training datasets were studied. The

544 first group includes only neural responses to pure noise (labeled as noise) and pure vocalization

545 (labeled as vocalization). The second group includes neural responses to $20 \mathrm{~dB}$ SNR as extra

546 training samples labeled as vocalization. The resulting classifier performances are displayed in

547 Figure 15. When only responses to pure noise and pure vocalization are used as training

548 samples, the performances under both noise conditions are not ideal, and greatly degrade around

$54915 \mathrm{~dB}$ SNR as shown in Figure 15A. However, the performance of classifiers trained by the

550 second group of neural responses shows an overall improvement. In Figure 15B, All the lines

551 shift towards the left, with smaller differences between vocalizations, and lead to a lower

552 detection threshold of around $5 \mathrm{~dB}$ SNR regardless of noise type. Therefore, by training on

553 neural responses contaminated by a small amount of noise, we can obtain classifiers with more

554 generalized performance over multiple SNR levels. 

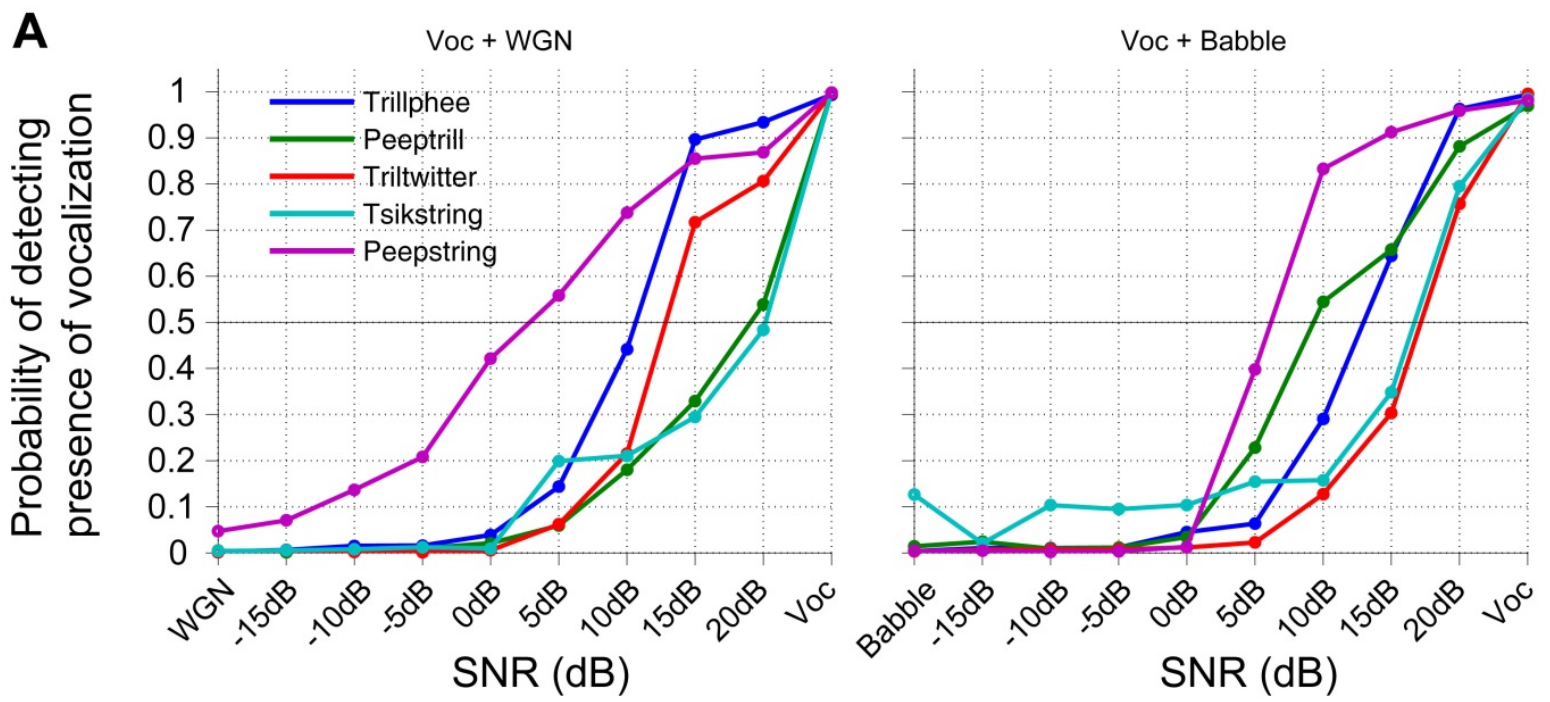

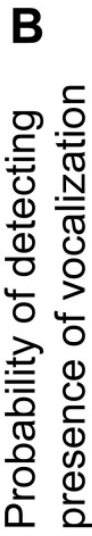

$V o c+20 d B+W G N$

1
0.9
0.8
0.7
0.6
0.5
0.4
0.3
0.2
0.1
0

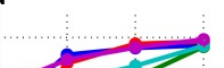

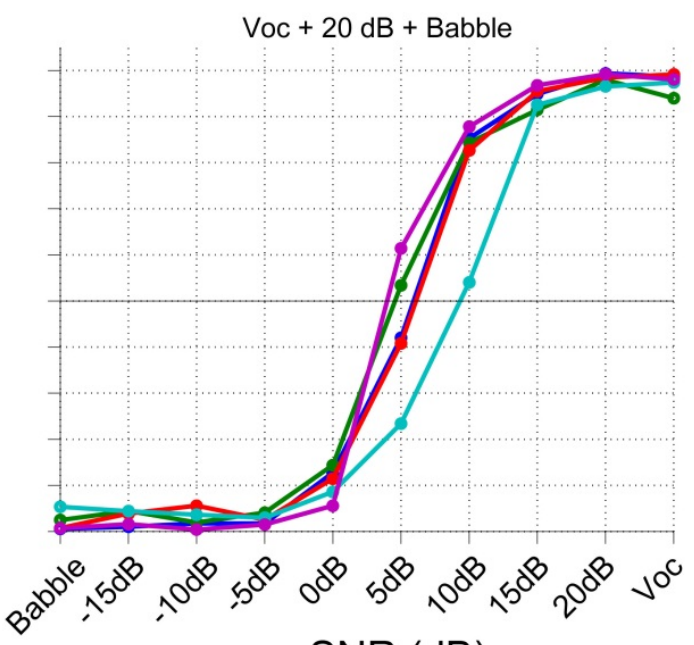

$\operatorname{SNR}(d B)$

\section{Figure 15: Noise enhances classifiers' detection threshold}

557 (A) Discriminability of population responses based on vocalizations and pure noises 558 (WGN/Babble).

559 (B) Discriminability of population responses based on vocalizations, 20 dB SNR noisy 560 vocalizations, and pure noises (WGN/Babble).

\section{Discussion [787/1500 words]}

We examined how the responses of a population of A1 neurons encode vocalizations at multiple intensities and SNR levels by studying the spatiotemporal structures of reduced population responses. We also investigated how well the combined responses of populations of neurons could be used to discriminate among vocalizations under different conditions. 
Temporally unstructured stimuli presentations have been demonstrated to induce neural codes of dynamic evolution (Bartho et al. 2009; Friedrich and Laurent 2001; Hegdé and Van

570 Essen 2004; Stopfer et al. 2003; Sugase et al. 1999). By projecting high-dimensional neural

571 responses into a lower dimensional space, we visualized the spatiotemporal structures of

572 population responses induced by complex stimuli: vocalizations at multiple intensities and

573 multiple SNR levels. Even though vocalizations delivered at different intensities are perceptually

574 similar, population responses were progressively differentiated with time, and produced finer

575 discrimination. Different vocalizations can be easily identified by their unique trajectories in

576 space. Some trajectories are relatively smooth and simple, while others are more convoluted, and

577 this variety is associated with the acoustic features of the vocalizations. Differentiation of

578 population response trajectories over time in an auditory scene was dependent on the noise type.

579 WGN noise led to more separable trajectories across SNR levels than Babble, and demonstrated

580 spatiotemporal analysis as a useful indicator of the difficulty of vocalization perception.

581 Consistent with the population coding of tone stimuli (Bartho et al. 2009), population response

582 vectors had the largest rotation during the initial hundreds of milliseconds in response to

583 vocalizations under different conditions, which is probably a common feature shared by

584 population responses to acoustic stimuli regardless of the complexity of stimuli. In addition, we

585 also implemented the same angle evolution analysis using raw population PSTH (data not

586 shown), and revealed a much weaker relationship between the angle evolution and vocalization

587 temporal envelope. This finding indicates that the information of vocalizations is well encoded in

588 a subset of neurons, as the reduced population responses are actually representations of partial

589 covariance in the whole population. 
Building neural response classifiers allowed us to investigate the optimal temporal

591 resolution and temporal dynamics of cortical detection and discrimination. Cortical

592 discrimination has been extensively studied for single units, and the optimal temporal resolution

593 was demonstrated to be 10 ms (Machens et al. 2003; Narayan et al. 2006; Rieke 1999; Schneider

594 and Woolley 2010). For our population of neurons, we also found that the temporal resolution

595 for cortical discrimination between vocalizations at multiple intensities on the population level

596 was optimized around $10 \mathrm{~ms}$. This time scale is small enough to capture temporal structures of

597 vocalization, and wide enough to allow integration of information over time, thereby reducing

598 noise. The temporal resolution for cortical discrimination between noisy vocalizations, however,

599 was smaller than $10 \mathrm{~ms}$. In our analysis, the best value was at $5 \mathrm{~ms}$, which is the finest temporal

600 resolution studied here. It is possible that the optimal temporal resolution for noisy vocalization

601 discrimination is below $5 \mathrm{~ms}$. A finer temporal resolution might reduce the interference of the

602 noise component in the auditory scene on vocalization recognition, because a longer time

603 window potentially introduces more noise information, thus confounding the vocalization

604 discrimination. The analysis of the temporal dynamics of discrimination revealed a range for the

605 time scale of integration on the order of hundreds of milliseconds, with $\sim 100 \mathrm{~ms}$ for

606 vocalizations at multiple intensities and $\sim 300 \mathrm{~ms}$ for noisy vocalizations. The time scale of

607 integration provides information about the speed of accumulation of discrimination accuracy at

608 the population level, and is in similar range to that of single units.

609 Whether information about sensory stimuli is best represented by the whole population of 610 neurons or a subpopulation of neurons was debated. The discrimination by subpopulations of

611 neurons was particularly studied for noisy vocalizations. We found that the subpopulation of the

612 robust group of neurons and the subpopulations of neurons excluding the brittle group both 
613 yielded slightly lower detection thresholds than the whole population, thus a better

614 discrimination performance. This result indicates that the brittle group of neurons contributes as

615 a neural distractor for noisy vocalization discrimination, and that information about vocalization

616 is better encoded by a subpopulation of neurons instead. We also built classifiers to demonstrate

617 that we can generalize the discrimination over lower SNR levels by using neural responses

618 contaminated by a little acoustic noise as training samples. While we are not saying that the brain

619 actually decodes vocalization information in the same way that our classifiers do, the results are

620 consistent with a previous psychoacoustic study that demonstrated that introducing weak noises

621 in perception improved the detection thresholds of target signals (Zeng et al. 2000).

622 In summary, we investigated our data with population analytic techniques and revealed

623 population response dynamics that cannot be fully evaluated by single-unit analysis alone. 
626 Figure 1: Acoustic stimuli used to investigate robust sound encoding in auditory cortex (A) Power spectrum of five vocalizations (solid lines), WGN (gray lines) and Babble noise (dashed lines). Background noises were truncated to have the same duration as each vocalization. The temporal waveform of each vocalization is displayed above each power spectrum. (B) Example spectrogram of vocalization Trillphee in noise at 10 different SNR levels, including pure noise and pure vocalization. The first column is Trillphee with WGN as background noise, and the second column is with Babble as background noise. The temporal waveform of WGN and Babble are shown below each column.

Figure 2: Trajectories of population responses to vocalizations at multiple intensities in 3D space

Figure 3: Evolution of rotation angles relative to the first time point (in silence) of the population response at multiple intensities in $3 \mathrm{D}$ space

Figure 4: Evolution of the rotation angles of population responses at multiple intensities relative to the population response at $75 \mathrm{~dB}$ SPL in $3 \mathrm{D}$ space

Figure 5: Population-averaged responses to five vocalizations in noise at multiple intensities in $3 \mathrm{D}$ response space

Figure 6: Intra-trajectory angle evolutions in 3D response space for noisy vocalization

Figure 7: Inter-trajectory angle evolutions in 3D response space for noisy vocalization

Figure 8: Discriminability of population spike trains in response to vocalizations at a variety of temporal resolutions

Figure 9: Dynamic discriminability of population spike trains in response to vocalizations over time

(A) Population discrimination of clean vocalizations based on single time bins evolving over time.

(B) Population discrimination of clean vocalizations based on increasing length of time bins evolving over time.

Figure 10: The influence of the number of neurons on population discriminability

(A) Classification accuracy of population responses of five clean vocalizations at different intensities.

(B) Confusion matrix of population responses classification.

Figure 11: Discriminability of population spike trains between pure noise (WGN/Babble)

666

667 
668 Figure 12: Discriminability of population spike trains between pure noise (WGN/Babble) 669 and noisy vocalizations at a variety of temporal resolutions

670 (A) Discriminability of population spike trains between pure noise (WGN/Babble) and noisy 671 vocalizations as a function of SNR.

672 (B) Confusion matrices of population response discriminability between pure noise 673 (WGN/Babble) and noisy vocalizations.

Figure 13: Dynamic discriminability of population spike trains in response to noisy vocalizations over time

677 (A) Population discrimination of noisy vocalizations based on single time bins evolving over 678 time.

679 (B) Population discrimination of noisy vocalizations based on increasing length of time bins 680 evolving over time.

Figure 14: Optimal sub-population for discrimination

(A) Performance of subset of robust neurons discriminating noisy vocalizations.

(B) Performance of subset of neurons including robust, balanced, and insensitive to discriminating noisy vocalizations.

Figure 15: Noise enhances classifiers' detection threshold

690 (B) Discriminability of population responses based on vocalizations, 20 dB SNR noisy

(A) Discriminability of population responses based on vocalizations and pure noises (WGN/Babble).

692

693 


\section{Reference}

696 Agamaite JA, Chang CJ, Osmanski MS, and Wang X. A quantitative acoustic analysis of the vocal repertoire of the common marmoset (Callithrix jacchus). J Acoust Soc Am 138: 2906-2928, 6982015.

Aggelopoulos NC, Franco L, and Rolls ET. Object perception in natural scenes: encoding by inferior temporal cortex simultaneously recorded neurons. J Neurophysiol 93: 1342-1357, 2005.

Aitkin LM, Merzenich MM, Irvine DR, Clarey JC, and Nelson JE. Frequency representation in auditory cortex of the common marmoset (Callithrix jacchus jacchus). J Comp Neurol 252: 175-185, 1986.

Anderson B, Sanderson MI, and Sheinberg DL. Joint decoding of visual stimuli by IT neurons' spike counts is not improved by simultaneous recording. Experimental brain research 176: 1-11, 2007.

Baeg E, Kim Y, Huh K, Mook-Jung I, Kim H, and Jung M. Dynamics of population code for working memory in the prefrontal cortex. Neuron 40: 177-188, 2003.

Barbour DL. Intensity-invariant coding in the auditory system. Neurosci Biobehav Rev 35: 2064-2072, 2011.

Barbour DL, and Wang X. Auditory cortical responses elicited in awake primates by random spectrum stimuli. The Journal of neuroscience 23: 7194-7206, 2003.

713 Bartho P, Curto C, Luczak A, Marguet SL, and Harris KD. Population coding of tone

714 stimuli in auditory cortex: dynamic rate vector analysis. Eur J Neurosci 30: 1767-1778, 2009.

Bender DA, Ni R, and Barbour DL. Spontaneous activity is correlated with coding density in primary auditory cortex. Journal of Neurophysiology 116: 2789-2798, 2016.

Bendor D, and Wang X. The neuronal representation of pitch in primate auditory cortex.

718 Nature 436: 1161-1165, 2005.

Bishop CM. Training with noise is equivalent to Tikhonov regularization. Neural Comput 7: 108-116, 1995.

Churchland MM, Yu BM, Cunningham JP, Sugrue LP, Cohen MR, Corrado GS, Newsome WT, Clark AM, Hosseini P, Scott BB, Bradley DC, Smith MA, Kohn A, Movshon JA, Armstrong KM, Moore T, Chang SW, Snyder LH, Lisberger SG, Priebe NJ, Finn IM, Ferster D, Ryu SI, Santhanam G, Sahani M, and Shenoy KV. Stimulus onset quenches neural variability: a widespread cortical phenomenon. Nature neuroscience 13: 369-378, 2010.

Friedrich RW, and Laurent G. Dynamic optimization of odor representations by slow temporal patterning of mitral cell activity. Science 291: 889-894, 2001. 
Gochin PM, Colombo M, Dorfman GA, Gerstein GL, and Gross CG. Neural ensemble coding in inferior temporal cortex. J Neurophysiol 71: 2325-2337, 1994.

732 Hegdé J, and Van Essen DC. Temporal dynamics of shape analysis in macaque visual area V2.

733 J Neurophysiol 92: 3030-3042, 2004.

Imig TJ, Irons WA, and Samson FR. Single-unit selectivity to azimuthal direction and sound pressure level of noise bursts in cat high-frequency primary auditory cortex. J Neurophysiol 63:

736 1448-1466, 1990.

Jolliffe I. Principal component analysis. Wiley Online Library, 2002.

Machens CK, Schütze H, Franz A, Kolesnikova O, Stemmler MB, Ronacher B, and Herz AV. Single auditory neurons rapidly discriminate conspecific communication signals. Nat Neurosci 6: 341-342, 2003.

McIlwain JT. Population coding: a historical sketch. Prog Brain Res 130: 3-7, 2001.

Meyers EM, Freedman DJ, Kreiman G, Miller EK, and Poggio T. Dynamic population coding of category information in inferior temporal and prefrontal cortex. J Neurophysiol 100:

744 1407-1419, 2008.

Nagarajan SS, Cheung SW, Bedenbaugh P, Beitel RE, Schreiner CE, and Merzenich MM. Representation of spectral and temporal envelope of twitter vocalizations in common marmoset primary auditory cortex. J Neurophysiol 87: 1723-1737, 2002.

Narayan R, Grana G, and Sen K. Distinct time scales in cortical discrimination of natural sounds in songbirds. J Neurophysiol 96: 252-258, 2006.

Ni R, Bender DA, Shaneshi AM, Gamble JR, and Barbour DL. Contextual Effects of Noise on Vocalization Encoding in Primary Auditory Cortex. Journal of Neurophysiology jn.

$75200476.02016,2016$.

Nikolić D, Haeusler S, Singer W, and Maass W. Temporal dynamics of information content carried by neurons in the primary visual cortex. In: Advances in neural information processing

755 systems2006, p. 1041-1048.

756 Panzeri S, Pola G, and Petersen RS. Coding of sensory signals by neuronal populations: the 757 role of correlated activity. The Neuroscientist 9: 175-180, 2003.

758 Rieke F. Spikes: exploring the neural code. MIT press, 1999.

759 Saha D, Leong K, Li C, Peterson S, Siegel G, and Raman B. A spatiotemporal coding 760 mechanism for background-invariant odor recognition. Nat Neurosci 16: 1830-1839, 2013.

761 Schneider DM, and Woolley SM. Discrimination of communication vocalizations by single 762 neurons and groups of neurons in the auditory midbrain. J Neurophysiol 103: 3248-3265, 2010. 
763 Stephan H, Baron G, and Schwerdtfeger WK. The brain of the common marmoset (Callithrix

764 jacchus): a stereotaxic atlas. Springer Science \& Business Media, 2012.

765 Stopfer M, Jayaraman V, and Laurent G. Intensity versus identity coding in an olfactory

766 system. Neuron 39: 991-1004, 2003.

767 Sugase Y, Yamane S, Ueno S, and Kawano K. Global and fine information coded by single

768 neurons in the temporal visual cortex. Nature 400: 869-873, 1999.

769 Van Gestel T, Suykens JA, Lanckriet G, Lambrechts A, De Moor B, and Vandewalle J.

770 Bayesian framework for least-squares support vector machine classifiers, gaussian processes, and

771 kernel Fisher discriminant analysis. Neural Comput 14: 1115-1147, 2002.

772 Wang L, Narayan R, Grana G, Shamir M, and Sen K. Cortical discrimination of complex

773 natural stimuli: can single neurons match behavior? J Neurosci 27: 582-589, 2007.

774 Woolley SM, Gill PR, and Theunissen FE. Stimulus-dependent auditory tuning results in

775 synchronous population coding of vocalizations in the songbird midbrain. J Neurosci 26: 2499-

$776 \quad 2512,2006$.

777 Zeng F-G, Fu Q-J, and Morse R. Human hearing enhanced by noise. Brain research 869: 251-

$778 \quad 255,2000$. 